\title{
A computational analysis of the long-term regulation of
}

\section{arterial pressure [version 1; peer review: 1 approved, 2}

\section{approved with reservations]}

\author{
Daniel A. Beard ${ }^{1}$, Klas H. Pettersen², Brian E. Carlson³, Stig W. Omholt4, \\ Scott M. Bugenhagen ${ }^{3}$ \\ ${ }^{1}$ Molecular and Integrative Physiology, University of Michigan, Ann Arbor, MI 48105, USA \\ ${ }^{2}$ Department of Mathematical and Technological Sciences, Norwegian University of Life Science, Oslo, Norway \\ ${ }^{3}$ Department of Physiology, Medical College of Wisconsin, Milwaukee, WI 53226, USA \\ ${ }^{4}$ Cardiac Exercise Research Group, Department of Circulation and Medical Imaging, NTNU Norwegian University of Science and \\ Technology, Trondheim, Norway
}

V1 First published: 08 Oct 2013, 2:208
https://doi.org/10.12688/f1000research.2-208.v1

Latest published: 06 Dec 2013, 2:208

https://doi.org/10.12688/f1000research.2-208.v2

\section{Abstract}

The asserted dominant role of the kidneys in the chronic regulation of blood pressure and in the etiology of hypertension has been debated since the 1970s. At the center of the theory is the observation that the acute relationships between arterial pressure and urine production-the acute pressure-diuresis and pressure-natriuresis curves-physiologically adapt to perturbations in pressure and/or changes in the rate of salt and volume intake. These adaptations, modulated by various interacting neurohumoral mechanisms, result in chronic relationships between water and salt excretion and pressure that are much steeper than the acute relationships. While the view that renal function is the dominant controller of arterial pressure has been supported by computer models of the cardiovascular system known as the "Guyton-Coleman model", no unambiguous description of a computer model capturing chronic adaptation of acute renal function in blood pressure control has been presented. Here, such a model is developed with the goals of: 1. capturing the relevant mechanisms in an identifiable mathematical model; 2 . identifying model parameters using appropriate data; 3. validating model predictions in comparison to data; and 4. probing hypotheses regarding the long-term control of arterial pressure and the etiology of primary hypertension. The developed model reveals: long-term control of arterial blood pressure is primarily through the baroreflex arc and the renin-angiotensin system; and arterial stiffening provides a sufficient explanation for the etiology of primary hypertension associated with ageing. Furthermore, the model provides the first consistent explanation of the physiological response to chronic stimulation of the baroreflex.

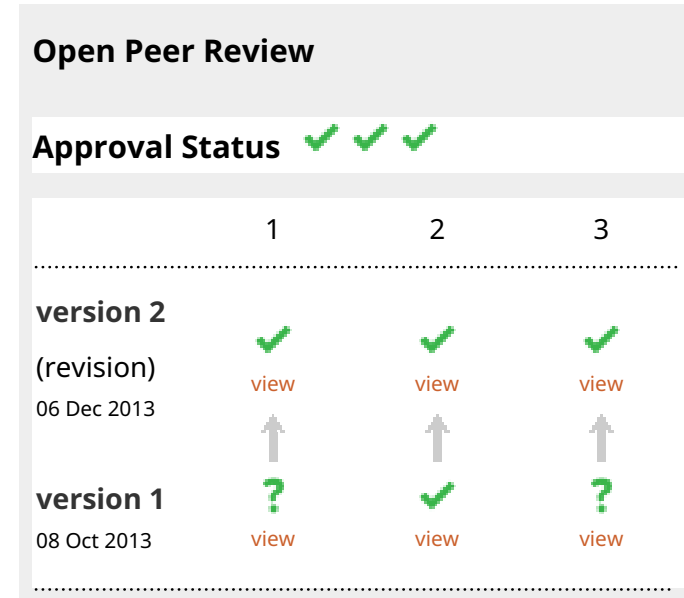

1. John Osborn, University of Minnesota, Minneapolis, MN, USA

Viktoria Averina, University of Minnesota, Minneapolis, MN, USA

2. Johnny Ottesen, Roskilde University, Roskilde County, Denmark

3. Patrick Hannaert, INSERM U1082, Poitiers, France

François Guillaud, INSERM U1082, Poitiers, France

Any reports and responses or comments on the article can be found at the end of the article. 
Corresponding author: Daniel A. Beard (beardda@umich.edu)

Competing interests: The authors have no competing interests to disclose.

Grant information: This work was funded by the VPR Project, supported by NIH grant P50GM094503. KHP is supported by the Research Council of Norway under the eVITA program, project number 178901/V30.

The funders had no role in study design, data collection and analysis, decision to publish, or preparation of the manuscript.

Copyright: $\odot 2013$ Beard DA et al. This is an open access article distributed under the terms of the Creative Commons Attribution License, which permits unrestricted use, distribution, and reproduction in any medium, provided the original work is properly cited. Data associated with the article are available under the terms of the Creative Commons Zero "No rights reserved" data waiver (CC0 1.0 Public domain dedication).

How to cite this article: Beard DA, Pettersen $\mathrm{KH}$, Carlson $\mathrm{BE}$ et al. A computational analysis of the long-term regulation of arterial pressure [version 1; peer review: 1 approved, 2 approved with reservations] F1000Research 2013, 2:208

https://doi.org/10.12688/f1000research.2-208.v1

First published: 08 Oct 2013, 2:208 https://doi.org/10.12688/f1000research.2-208.v1 


\section{Introduction}

Theoretical analysis and observations of the control of blood volume and salt content by the kidneys has led to the hypothesis that arterial pressure is determined in the long-term (over time scales of days or more) by the balance between the level of salt intake and the acute relationship between pressure and salt excretion by the kidneys. In fact, it is stated that the renal pressure/volume control system adjusts arterial pressure with "infinite gain" and that the renal function curve and rate of salt and water intake are the "two

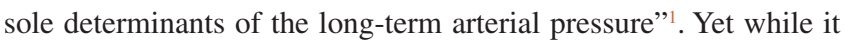
is certain that at any steady level of arterial pressure the rates of salt and water intake and excretion are balanced, there is considerable debate over how this balance is achieved, and thus what are the long-term determinants of arterial pressure ${ }^{2-5}$.

One viewpoint is that the acute relationship between pressure and salt excretion - the acute pressure-natriuresis mechanism-represents a physiological input-output relationship and that alterations to this mechanism underlie most (if not all) chronic changes in pressure ${ }^{1,6}$. While competing ideas identify the nervous system and its influence on the heart and vasculature as the primary long-term controllers of arterial pressure ${ }^{3,4}$, the hypothesis that long-term control of blood pressure is achieved through the renal pressure-diuresis represents the dominant the thinking in the field ${ }^{7}$.

The most widely recognized model of long-term blood pressure control is the model developed over several decades by Guyton and colleagues. The 1972 realization of the model ${ }^{6}$ invokes approximately 160 variables, and several hundred adjustable parameters $^{8}$. More recent versions of the model involve many thousands of variables and tens of thousands of parameters'. While several realizations of the Guyton model are or have been disseminated as computer programs, human readable expositions of the various versions of the model are lacking. Moreover, there is no published report of any of these models in which the governing equations are defined, the parameter values are provided, the data that were used for model identification are reported, and the model identification process and results are reported. Indeed, it is unlikely if not impossible that even the relatively simple 1972 version of the model was formally identified. These facts unfortunately make this seminal work largely impenetrable and possibly irreproducible. Most critically, the Guyton-Coleman model is based on the assertion that arterial blood pressure is, under all circumstances, controlled primarily by the kidney ${ }^{10}$. Therefore the Guyton-Coleman model cannot be used to explore alternative hypotheses.

In the Systems Approach for PHysiological Integration of Renal, cardiac and respiratory functions (SAPHIR) project, Thomas et $a .^{8}$ aimed to develop and disseminate a core "model of human physiology targeting the short- and long-term regulation of blood pressure, body fluids and homeostasis of the major solutes" including "the main regulatory sensors (baro- and chemoreceptors) and nervous (autonomic control) and hormonal regulators (antidiuretic hormone, aldosterone and angiotensin)". The project goal is to, for the first time, provide an open-source transparent model integrating these systems. In 2008, the SAPHIR group published a core model of circulation and volume exchange across fluid compartments that is derived from the Guyton models, consisting of 20 equations and perhaps less than 100 parameters $^{8}$. This model does not account for adaptation of the acute pressure-diuresis/natriuresis function to chronic changes in arterial pressure or volume/salt loading. Thus, it does not capture the concept of the chronic renal function curve, which is thought to be of fundamental importance to the long-term regulation of arterial pressure. Recently Averina et $a l .{ }^{4}$ reported a mathematical model of long-term control of arterial pressure that captures the concept of chronic adaptation of pressure-natriuresis and, to our knowledge, represents the only published model incorporating the concept of adaptation of the acute pressure-natriuresis relationship in response to changes in salt/volume loading. Thus, the model of Averina et al. represents the model of record of regulation of blood pressure accounting for the chronic renal function curve. Yet, this model was developed to illustrate that the chronic renal function curve need not represent a physiological input-output mechanism, and to introduce an alternative description of the long-term regulation of arterial pressure and the etiology of salt- and angiotensindependent hypertension.

Similar to the goals of the SAPHIR project, we developed a mathematical model of the Guyton concept of long-term control of arterial pressure, analysed its behavior and compared its predictions to experimental observations. Similarly, an open-source transparent model integrating these systems is provided. The approach pursued here differs from that of Thomas and colleagues in that while the SAPHIR project explicitly centers on translating the components of the Guyton model into an explicitly defined core model, our goal is to develop a model from scratch in which all model components and associated data are formally identified based on experimental data. Furthermore, given the demands of model identification and validation, our goals are necessarily more narrowly constrained than those of the SAPHIR project and of the original Guyton-Coleman models. In our study, a model is constructed in the spirit of the Guyton-Coleman models, adopting a practical approach to datadriven phenomenological representations of physiological systems, rather than aiming for physical and mechanistic realism.

Specifically, we have developed a model of the long-term control of arterial pressure that captures the Guyton concept of pressurediuresis/natriuresis as physiological input-output relationships. The model is designed to meet the following criteria: 1 . The model accounts for the effects of the baroreflex on the circulation, the heart, and the kidney, and the effects of the renin-angiotensin system on the circulation, the heart, and the kidney; 2 . The model is explicitly documented for definition and reproducibility: all model equations are reported herein and justified based on experimental data; 3 . Parameter values are reported and justified: All parameter values are estimated by comparing model simulations to measured data. The identified model is used to probe physiological mechanisms underlying the chronic renal function curve, to provide insight into how chronic stimulation of the baroreflex leads to chronic reductions in arterial pressure, and to generate hypotheses regarding the etiology of primary hypertension.

\section{Methods}

Model Components 1: Aorta/large-artery mechanics

Based on the simple approximation of a thin-walled cylinder, the strain $\varepsilon$ in the aorta is computed as a function of volume $V_{A o}$ 


$$
\varepsilon=\frac{d_{A}}{d_{0}}=\left(\frac{V_{A o}}{V_{0}}\right)^{1 / 2},
$$

where $V_{0}$ is a parameter representing the unstressed volume, and $d_{A} / d_{0}$ is the ratio of diameter to unstressed diameter. (Here $\varepsilon$ is defined to be equal to 1 in the unstressed state when $d_{A}=d_{0}$ ). The aortic pressure-volume relationship is assumed to be governed by

$$
P_{A o}=\left(V_{A o}-V_{s A o}\right) / C_{A o},
$$

where $C_{A o}$ represents and acute compliance and $V_{s A o}(t)$ accounts for creep mechanics of aortic wall, simulated according to

$$
\begin{aligned}
& \tau_{c A o} \frac{d V_{s A o}}{d t}=V_{s A o}^{\infty}-V_{s A o} \\
& V_{s A o}^{\infty}=\left(1-\frac{C_{A o}}{C_{\infty}}\right) V_{A o}=\gamma_{A o} V_{A o},
\end{aligned}
$$

where $\tau_{c A o}$ is the time constant of stress relaxation and $C_{A o} / C_{\infty}$ is the ratio of acute to effective chronic compliance of the vessel. (Equation (2) and Equation (3) represent an alternative equivalent formulation of the standard linear model of vessel mechanics).

The parameters of the large-artery mechanics model component are identified based on measurement of aortic diameter and pressure in dogs reported by Coleridge et al. ${ }^{11}$. Figure 1 plots the measured aortic pressure wave in the upper panel and vessel diameter as a function of pressure in the lower. The experiment of Coleridge et al. is simulated using the governing equations

$$
\begin{aligned}
& \tau_{c A o} \frac{d V_{s A o}}{d t}=V_{s A o}^{\infty}-V_{s A o} \\
& \frac{d V_{A o}}{d t}=C_{A o} \frac{d P_{A o}}{d t}+\frac{d V_{s A}}{d t} \\
& \frac{d \varepsilon}{d t}=\frac{1}{2\left(V_{A o} V_{0}\right)^{1 / 2}} \frac{d V_{A o}}{d t},
\end{aligned}
$$

where the measured aortic pressure waveform (Figure 1A) is used to numerically approximate $d P_{A 0}(t) / d t$ in integrating Equation (4).

The parameters $\gamma_{A o}, C_{A o}, \tau_{c A}$, and $d_{0}$ were adjusted to match the data in Figure 1B; the value of $V_{0}$ was set arbitrarily by assuming a cylindrical vessel of length $30 \mathrm{~mm}$. All parameter values are listed in Table 1 .

Model Components 2: Kinetics of baroreflex afferent firing The baroreceptor afferent firing rate is assumed to be governed by the rate of change of strain in the vessel wall. The model invokes a moving average strain value $\bar{\varepsilon}(t)$, which is computed

$$
\tau_{s} \frac{d \bar{\varepsilon}}{d t}=\varepsilon-\bar{\varepsilon} .
$$

The time constant $\tau_{s}$ is an adjustable parameter. The baroreceptor firing rate is assumed proportional to $\delta_{\varepsilon}=\max (\varepsilon-\bar{\varepsilon}, 0)$ via the saturable relationship

$$
f_{B R}(t)=f_{0} s(t) \frac{\delta_{\varepsilon}(t)}{\delta_{\varepsilon}(t)+\delta_{0}},
$$

where $s(t)$ represents the fraction of baroreceptor afferents that are in an active/permissible state, and $\delta_{\varepsilon}$ and $f_{0}$ are additional adjustable parameters. Equation (6) is a static nonlinearity ${ }^{12}$ that enforces a saturating response. It is assumed that the baroreceptors within the population transition from an active to inactive state at a rate proportional to the firing rate, and transition to the active state at a constant rate:

$$
\frac{d s}{d t}=a(1-s)-b s \frac{\delta_{\varepsilon}}{\delta_{\varepsilon}+\delta_{0}} .
$$

The adjustable parameters in the baroreflex afferent model $\left(\tau, \delta_{\varepsilon}\right.$, $f_{0}, a$, and $b$ ) are identified based on measurements following step changes in non-pulsatile carotid sinus pressure ${ }^{13}$ and ramps in in vivo pulsatile aortic pressure ${ }^{11}$.
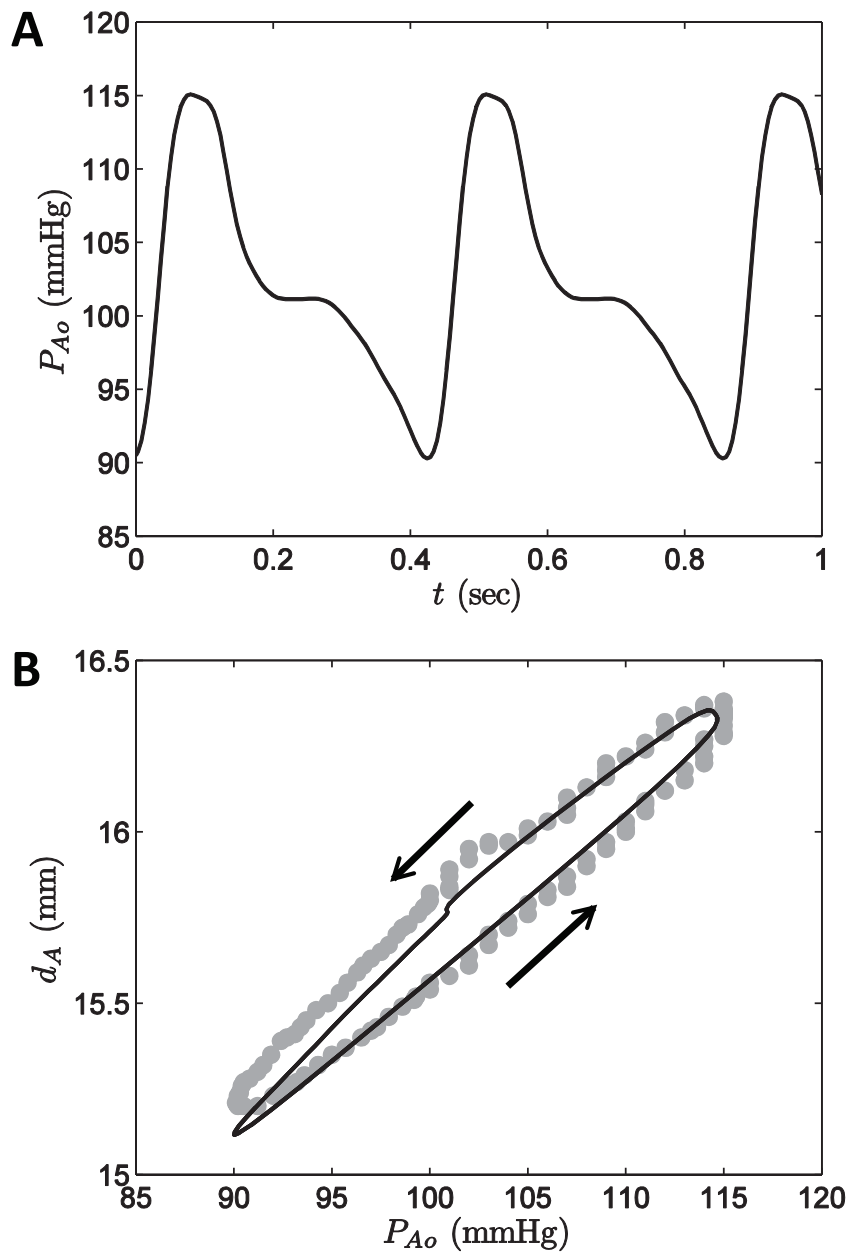

Figure 1. Simulated aortic mechanics. A. The aortic pressure time course obtained from Coleridge et al. ${ }^{11}$ is used as the input to the aortic mechanics model module, Equation (4). B. The modelpredicted relationship between aortic pressure and diameter is compared to the data reported by Coleridge et al. ${ }^{11}$. Model simulations are plotted as a solid black cure; data are plotted as shaded circles. 


\section{Table 1. Model parameters.}

\begin{tabular}{|c|c|}
\hline \multicolumn{2}{|l|}{ Aorta/large-artery mechanics } \\
\hline$V_{0}=0.6875 \mathrm{ml}$ & Aortic mechanics, Equation (1) \\
\hline$d_{0}=12 \mathrm{~mm}$ & Aortic mechanics, Equation (1) \\
\hline$C_{A o}=0.007 \mathrm{ml} \mathrm{mmHg}^{-1}$ & Aorta acute capacitance, Equation (2) \\
\hline$\gamma_{A o}=0.40$ & Aorta creep parameter, Equation (3) \\
\hline$\tau_{c A o}=0.12 \mathrm{sec}$ & Aorta creep time constant, Equation (3) \\
\hline \multicolumn{2}{|l|}{ Kinetics of baroreflex afferent firing } \\
\hline$\tau_{s}=251.5 \mathrm{sec}$ & Baroreceptor parameter, Equation (5) \\
\hline$a=0.0651 \mathrm{sec}^{-1}$ & Baroreceptor activation rate, Equation (7) \\
\hline$b=0.2004 \mathrm{sec}^{-1}$ & Baroreceptor deactivation rate, Equation (7) \\
\hline$\delta_{0}=0.4965$ & Baroreceptor saturation constant, Equation (7) \\
\hline$f_{0}=299.8 \mathrm{sec}^{-1}$ & Baroreceptor gain parameter, Equation (6) \\
\hline \multicolumn{2}{|c|}{ Mechanics of the heart and circulation } \\
\hline$E_{\max }=8 \mathrm{mmHg} \mathrm{ml}^{-1}$ & Varying elastance heart model, Equation (10) \\
\hline$E_{\min }=0.25 \mathrm{mmHg} \mathrm{ml}^{-1}$ & Varying elastance heart model, Equation (10) \\
\hline$T_{M}=0.3$ & Varying elastance heart model, Equation (10) \\
\hline$T_{R}=0.15$ & Varying elastance heart model, Equation (10) \\
\hline$H_{0}=75$ beat $\mathrm{min}^{-1}$ & Varying elastance heart model, Equation (11) \\
\hline$H_{1}=100$ beat $\mathrm{min}^{-1}$ & Varying elastance heart model, Equation (11) \\
\hline$R_{\text {out }}=1 \times 10^{-4} \mathrm{mmHg} \mathrm{min} \mathrm{ml}^{-1}$ & Aortic valve resistance, Equation (12) \\
\hline 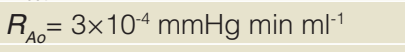 & Aortic resistance \\
\hline$R_{A 0}=0.01234 \mathrm{mmHg} \mathrm{min} \mathrm{ml}^{-1}$ & Large-artery resistance, Equation (12) and Equation (13) \\
\hline 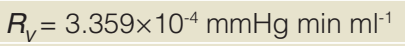 & Downstream (venous) resistance, Equation (12) \\
\hline$C_{A 0}=0.8174 \mathrm{ml} \mathrm{mmHg}^{-1}$ & Large-artery compliance, Equation (13) \\
\hline$C_{v_{0}}=329.45 \mathrm{ml} \mathrm{mmHg}^{-1}$ & Downstream (venous) resistance, Equation (13) \\
\hline$V_{v 01}=625.1 \mathrm{ml}$ & Unstressed volume of cardiovascular system, Equation (14) \\
\hline$\gamma_{v}=0.40$ & Venous creep parameter, Equation (15) \\
\hline$\tau_{c v}=120 \mathrm{sec}$ & Venous creep time constant, Equation (15) \\
\hline$\alpha_{1}=0.319$ & Arterial and venous compliance parameter, Equation (13) \\
\hline$\alpha_{2}=12.77$ & Arterial resistance parameter, Equation (13) \\
\hline$\alpha_{3}=1.027$ & Arterial and venous compliance parameter, Equation (13) \\
\hline$\alpha_{4}=2.972$ & Arterial resistance parameter, Equation (13) \\
\hline$F_{0}=1125 \mathrm{ml} \mathrm{min}^{-1}$ & Autoregulation parameter, Equation (16) \\
\hline$F_{1}=487.2 \mathrm{ml} \mathrm{min}^{-1}$ & Autoregulation parameter, Equation (16) \\
\hline$\tau_{A R}=6.455 \mathrm{~min}$ & Autoregulation parameter, Equation (16) \\
\hline$\tau_{F}=15 \mathrm{sec}$ & Autoregulation parameter, Equation (17) \\
\hline \multicolumn{2}{|l|}{ Autonomic system } \\
\hline$f_{S N}=2.76 \mathrm{sec}^{-1}$ & Baroreflex arc parameter, Equation (18) \\
\hline \multicolumn{2}{|l|}{ Renin-angiotensin system } \\
\hline$\tau_{R}=12.59 \mathrm{~min}$ & Time constant for renin production, Equation (19) \\
\hline$\tau_{A 2}=1.065 \mathrm{~min}$ & Time constant for angiotensin II production, Equation (20) \\
\hline$\tau_{p}=15 \mathrm{sec}$ & Time constant for mean pressure calculation, Equation (19) \\
\hline$P_{1}=19.18 \mathrm{mmHg}$ & Steady-state renin-angiotensin system tone, Equation (19) \\
\hline$P_{2}=25.0 \mathrm{mmHg}$ & Steady-state renin-angiotensin system tone, Equation (19) \\
\hline$g=246.6 \mathrm{mmHg}$ & Steady-state renin-angiotensin system tone, Equation (19) \\
\hline \multicolumn{2}{|c|}{ Neurohumoral control of pressure-diuresis/natriuresis } \\
\hline$k_{1}=0.125 \mathrm{ml} \mathrm{sec}^{-1} \mathrm{mmHg}^{-1}$ & Slope of acute pressure-diuresis relationship, Equation (21) \\
\hline$P_{s, \max }=126.4 \mathrm{mmHg}$ & Long-term pressure-diuresis relationship, Equation (22) \\
\hline$P_{s, \min }=9.779 \mathrm{mmHg}$ & Long-term pressure-diuresis relationship, Equation (22) \\
\hline$\phi_{0}=0.1928$ & Long-term pressure-diuresis relationship, Equation (22) \\
\hline$\phi_{1}=0.4813$ & Long-term pressure-diuresis relationship, Equation (22) \\
\hline$\tau_{k}=10 \mathrm{~min}$ & Time constant for long-term pressure-diuresis, Equation (22) \\
\hline
\end{tabular}


The data in Figure 2A, obtained from Chapleau et al. ${ }^{13}$, correspond to the multi-fiber response of the carotid sinus baroreceptors following a step change in pressure from $40 \mathrm{mmHg}$ to $93 \mathrm{mmHg}$. To simulate this experiment, the pressure is assumed to follow a time course described by

$$
P_{A o}(t)=\left\{\begin{array}{l}
40, t<-1 \\
40+53 \cdot(t+1), t \geq-1, \\
93, t \geq 0
\end{array}\right.
$$

where pressure is expressed in units of $\mathrm{mmHg}$ and time in seconds. Thus it is assumed that pressure is increased by $+53 \mathrm{mmHg}$ over an interval from -1 to 0 seconds. Since the overall model does not differentiate between aortic and carotid baroreflex signals, data from the carotid pressure step are matched to the model of Equation (4) -Equation (7) in Figure 2A. Following the step pressure increase the afferent firing rate $f_{B R}$ rapidly increases to a maximum value before decaying over a time scale of several hundred seconds. This decay is determined in the model by the combined action of mechanical relaxation governed by Equation (5) and inactivation governed by Equation (7).
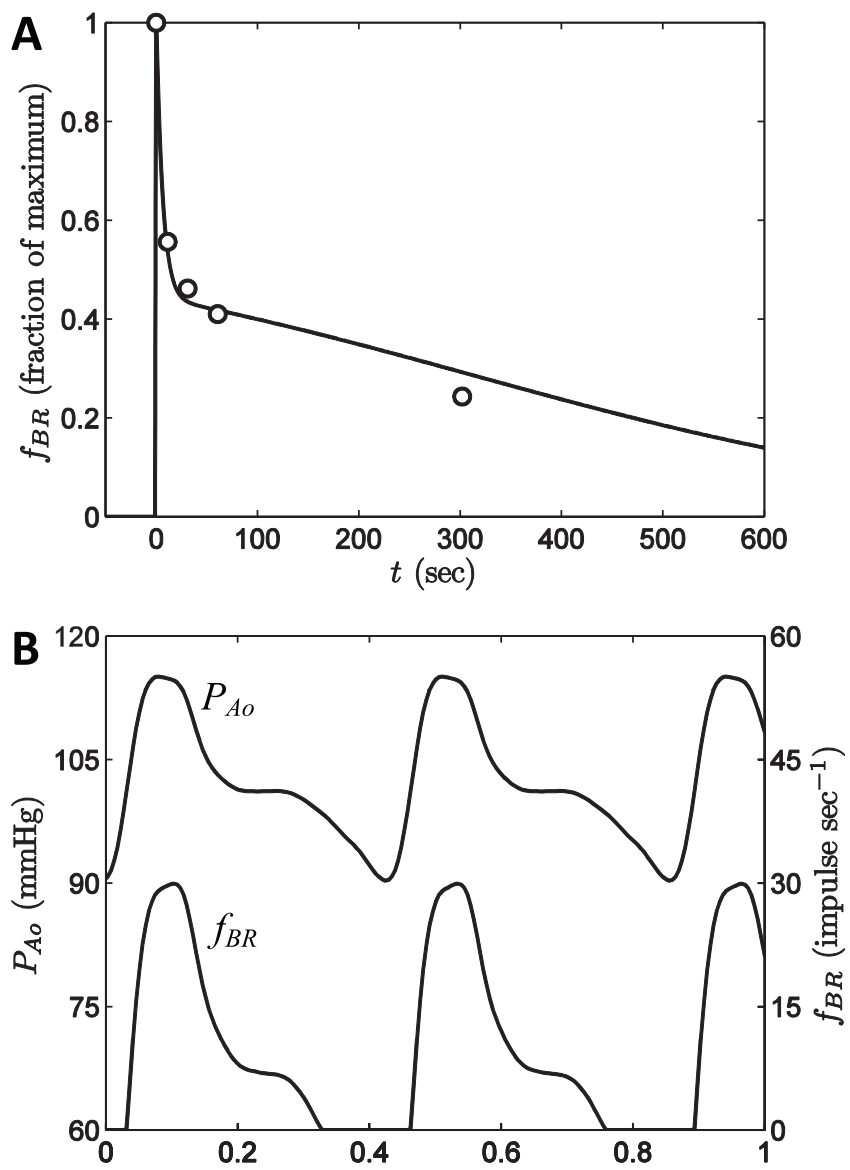

Figure 2. Baroreflex model. A. The response of the baroreflex model to a step increase in pressure is compared to data from Chapleau et al..$^{13}$. The model simulations are based on Equation (4)-Equation (7), as described in the text. B. Simulated baroreflex output based on input aortic pressure wave obtained from Coleridge et al. ${ }^{11}$.
Figure 2B plots model-predicted baroreflex firing rate elicited by the normal aortic pressure waveform of Coleridge et al. ${ }^{11}$. The model produces the characteristic bursting pattern where peaks in baroreceptor afferent firing occur in systole, with the firing rate dropping to zero in diastole.

Responses to ramps of pressure in vivo are compared to data of Coleridge et al. in Figure 3. Coleridge et al. adjusted in vivo pressure in the aortic sinus by placing hydraulic occlusion at various positions along the aorta. Model simulations are based on applying a constant increase/decrease to measured $P_{A o}(t)$, resulting in the pressure time series plotted in Figure $3 \mathrm{~A}$ and $3 \mathrm{C}$. It is assumed that mean pressure is adjusted at a rate of $\pm 10 \mathrm{mmHg} \mathrm{sec}{ }^{-1}$, corresponding to the experimental measurements. The top panel in the figure corresponds to simulations and data associated with the baseline state, where initial mean pressure is $100 \mathrm{mmHg}$. Data on afferent firing rate for this experiment are plotted as open circles in Figure 3B. The solid line in Figure $3 \mathrm{~B}$ represent simulated $f_{B R}(t)$ versus $P_{A o}(t)$. The red line represents $f_{B R}(t)$ and $P_{A o}(t)$ averaged over each beat. The lower panel in Figure 3 corresponds to a hypertensive state where the aortic pressure was held at an elevated level with mean of $125 \mathrm{mmHg}$ for 20 minutes prior to the pressure ramp experiment. Thus the system has had 20 minutes to adapt or "reset" to a new mean pressure, resulting in a right shift of the baroreflex response $f_{B R}$ versus $P_{A 0}$.

The values of the parameters $\tau_{s}, \delta_{0}, f_{0}, a$, and $b$ were adjusted to match the data in Figure 2A and Figure 3.

\section{Model Components 3: Mechanics of the heart and circulation}

The circulation is modeled as a closed-loop lumped-parameter circuit illustrated in Figure 4, which ignores the pulmonary circulation and treats the heart as time-varying elastance representing the left ventricle $^{14}$. The left-ventricular pressure is described by

$$
P_{L V}(t)=E_{L V}(t) \cdot V_{L V}(t),
$$

where $E_{L V}(t)$ is the left-ventricular elastance and $V_{L V}(t)$ is the volume of blood in the ventricle. The elastance is simulated using a smooth function that increases in systole and decreases as the heart relaxes:

$$
E_{L V}(\theta)= \begin{cases}\frac{\left(\left(0.75+\phi_{S V}\right) E_{\max }-E_{\min }\right)}{2}\left[1-\cos \left(\frac{\pi \theta}{T_{M}}\right)\right]+E_{\min } & 0 \leq \theta \leq T_{M} \\ \frac{\left(\left(0.75+\phi_{S N}\right) E_{\max }-E_{\min }\right)}{2}\left[\cos \left(\frac{\pi\left(\theta-T_{M}\right)}{T_{R}}\right)+1\right]+E_{\min } & T_{M} \leq \theta \leq T_{M}+T_{R}, \\ E_{\min } & T_{M}+T_{R} \leq \theta \leq 1\end{cases}
$$

where $\theta \in(0,1)$ is the fraction of a heart beat that has elapsed at a given time. The factor $\left(0.75+\phi_{S N}\right)$ multiplying $E_{\max }$ accounts for the effect of sympathetic tone on contractility, where $\phi_{S N}(t) \in(0,1)$ is a model variable (see below) representing the sympathetic drive. Under baseline conditions $\phi_{S N} \approx 0.25$ and therefore, under maximal sympathetic stimulation, cardiac contractility is approximately $175 \%$ of baseline.

The variable $\theta$ is simulated via 

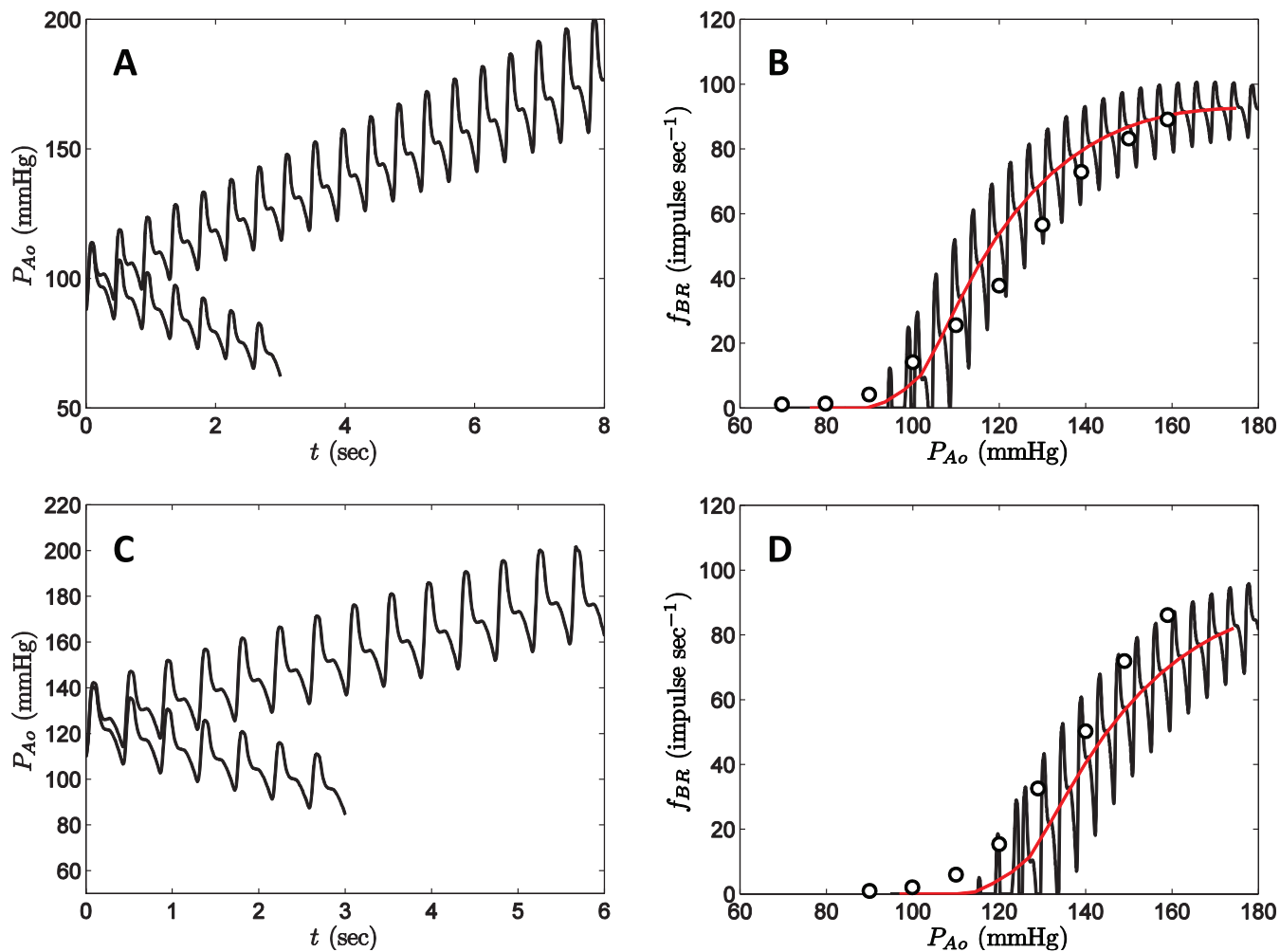

Figure 3. Baroreflex response to in vivo pressure ramps. A. Applied pressure transients for increasing and decreasing pressure starting at a baseline mean pressure of $100 \mathrm{mmHg}$. Pressure is increased/decreased so that the mean pressure changes at the rate of $\pm 10 \mathrm{mmHg}$ $\mathrm{sec}^{-1}$. B. Simulated baroreceptor firing response to pressure ramps from $\mathbf{A}$ is compared to data from Coleridge et al. ${ }^{11}$. C. Applied pressure transients for increasing and decreasing pressure starting at a baseline mean pressure of $125 \mathrm{mmHg}$. D. Simulated baroreceptor firing response to pressure ramps from $\mathbf{C}$ is compared to data from Coleridge et al. ${ }^{11}$. See text for details on simulation protocol. In $\mathbf{B}$ and D, experimental data are plotted as circles; black line represents the simulation prediction; red line represents simulation predictions averaged over each heart beat.

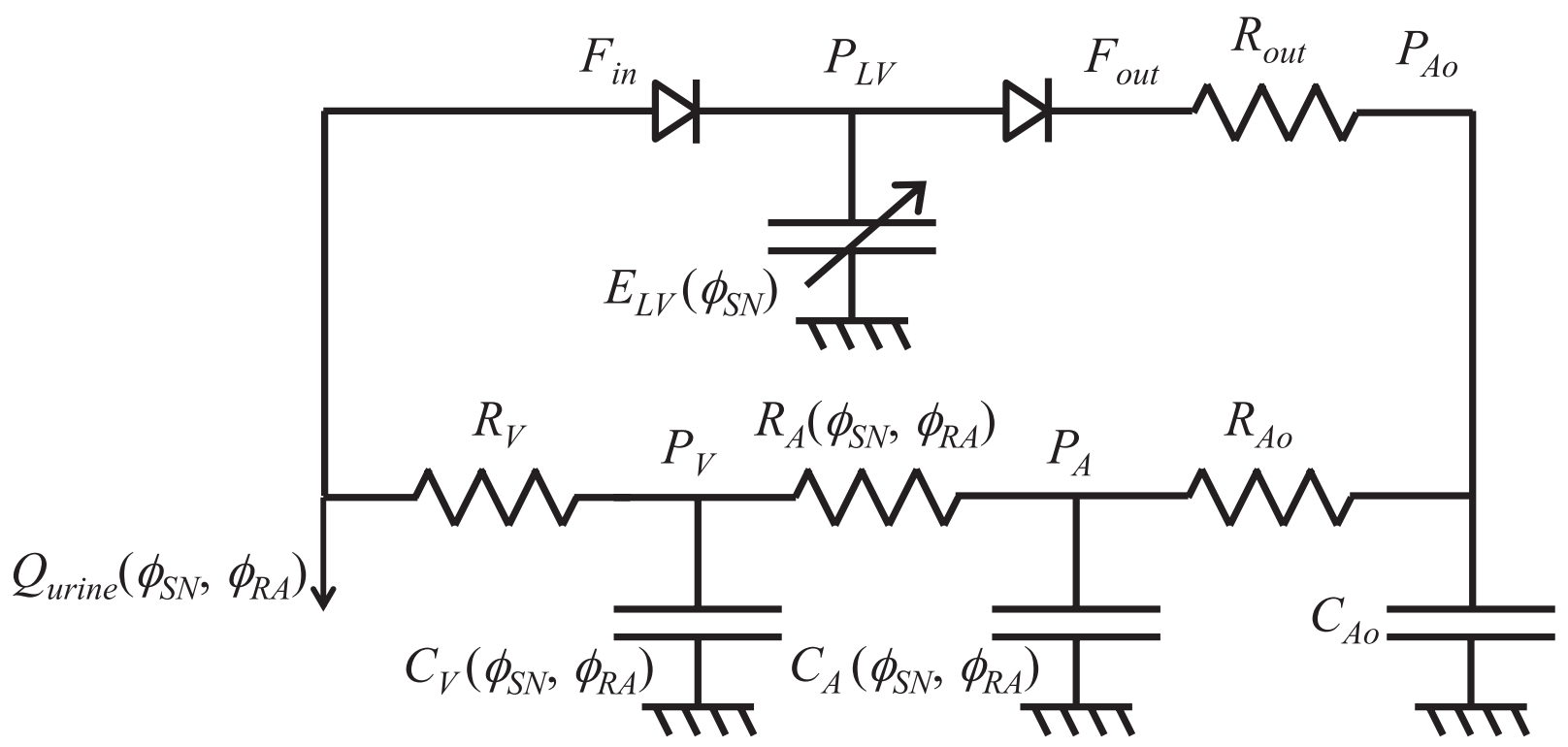

Figure 4. Diagram of cardiovascular circuit model representing the systemic circulation. Flows entering and exciting the heart are denoted $F_{\text {in }}$ and $F_{\text {out }}$; pressure in the aorta and arterial and venous capacitors are denoted $P_{A 0}, P_{A}$ and $P_{V}$; left-ventricular pressure is denoted $P_{L V}$ 


$$
\frac{d \theta}{d t}=H
$$

where $H=H_{0}+H_{1}\left(\phi_{S N}-0.25\right)$ is the heart rate, and $\theta(t)$ is reset to 0 each time the value reaches 1 . The parameters $H_{0}$ and $H_{1}$ are set to give a baseline heart rate of 75 beats $\min ^{-1}$ and a heart rate of 150 beats min $^{-1}$ under maximal sympathetic tone.

The circuit model is simulated based on equations for the state variables $\theta(t), V_{L V}(t), V_{A o}(t), V_{A}(t), V_{V}(t), V_{s A}(t)$, and $V_{s V}(t)$. The governing equations for the six volume variables are

$$
\begin{aligned}
& \frac{d V_{L V}}{d t}=\max \left(0, \frac{P_{V}-P_{L V}}{R_{V}}\right)-\max \left(0, \frac{P_{L V}-P_{A o}}{R_{\text {out }}}\right), \\
& \frac{d V_{A o}}{d t}=\max \left(0, \frac{P_{L V}-P_{A o}}{R_{\text {out }}}\right)-\frac{P_{A o}-P_{A}}{R_{A o}}, \\
& \frac{d V_{A}}{d t}=\frac{P_{A o}-P_{A}}{R_{A o}}-\frac{P_{A}-P_{V}}{r_{A R} R_{A}}, \\
& \frac{d V_{V}}{d t}=\frac{P_{A}-P_{V}}{r_{A R} R_{A}}-\max \left(0, \frac{P_{V}-P_{L V}}{R_{V}}\right)+Q_{\text {input }}-Q_{\text {urine }}, \\
& \frac{d V_{S A o}}{d t}=\left(V_{s A o}^{\infty}-V_{s A o}\right) / \tau_{c A o}, \\
& \frac{d V_{s V}}{d t}=\left(V_{s V}^{\infty}-V_{s V}\right) / \tau_{c V},
\end{aligned}
$$

where $P_{L V}(t)$ is determined by Equation (9).

The flows $Q_{\text {in }}$ and $Q_{\text {urine }}$ represent the rates of volume uptake/infusion and urine production, described below.

The $\max (0, \cdot)$ terms in Equation (12) account for the valves, which permit flow only in the direction indicated in Figure 4.

The $R_{A o}$ and $R_{V}$ resistances are set to constant values, while other resistances and capacitances vary with sympathetic tone and angiotensin II level. Specifically, $C_{A}(t), C_{V}(t)$, and $R_{A}(t)$ are determined by

$$
\begin{aligned}
& C_{A}(t)=\frac{C_{A 0}}{\left(1+\alpha_{1} \phi_{S N}(t)\right)\left(1+\alpha_{3} \phi_{A 2}(t)\right)} \\
& C_{V}(t)=\frac{C_{V 0}}{\left(1+\alpha_{1} \phi_{S N}(t)\right)\left(1+\alpha_{3} \phi_{A 2}(t)\right)} \\
& R_{A}(t)=R_{A 0}\left(1+\alpha_{2} \phi_{S N}(t)\right)\left(1+\alpha_{4} \phi_{A 2}(t)\right),
\end{aligned}
$$

where $C_{A 0}, C_{V 0}$, and $R_{A 0}$ are constants, and $\alpha_{1}, \alpha_{2}, \alpha_{3}$, and $\alpha_{4}$ are constants that determine the magnitude of the effects of vasoconstriction via sympathetic tone and angiotensin II. The variable $\phi_{A 2}$ represents the plasma angiotensin II activity. The governing equations for $\phi_{A 2}$ are described below.

The $R_{A}$ resistance is further governed by a whole-body autoregulation phenomenon, as incorporated into the Guyton-Coleman models ${ }^{6,8,9}$. The function $r_{A R}(t) \in(0,1)$ accounts for autoregulatory effects on systemic arterial conductivity based on

$$
\begin{aligned}
& \tau_{A R} \frac{r_{A R}}{d t}=r_{A R}^{\infty}-r_{A R} \\
& r_{A R}^{\infty}=\frac{1}{2}\left(1+\tanh \left(\frac{\bar{F}-F_{0}}{F_{1}}\right)\right)
\end{aligned}
$$

where $\bar{F}(t)$ is a variable that averages the mean cardiac output with a moving average defined by a first-order process:

$$
\tau_{F} \frac{d \bar{F}}{d t}=\frac{P_{A}-P_{V}}{r_{A R} R_{A}}-\bar{F} .
$$

The pressures are computed from the relationships

$$
\begin{aligned}
& P_{A o}=\left(V_{A o}-V_{s A}\right) / C_{A o} \\
& P_{A}=V_{A} / C_{A} \\
& P_{V}=\left(V_{V}-V_{s V}-V_{V 01}\right) / C_{V},
\end{aligned}
$$

in which venous compliance is simulated using a linear formulation of stress relaxation similar to that used for the aorta. Specifically, venous stress relaxation kinetics are governed by $\tau_{c V}\left(V_{s V} / d t\right)=V_{s V}^{\infty}-V_{s V}$, where

$$
V_{s V}^{\infty}=\left(1-\frac{C_{V}}{C_{\infty}}\right)\left(V_{V}-V_{V 01}\right)=\gamma_{V}\left(V_{V}-V_{V 01}\right)
$$

The constant $V_{V 01}$ in Equation (17) represents an unstressed volume for the overall cardiovascular system.

There are a total of 23 parameters associated with this component of the model. Assignment of the values listed in Table 1 was guided by a variety of data sets and previous computational models. The values of $E_{\max }$ and $E_{\max }$ were obtained by scaling the model of ${ }^{14}$ to provide reasonable pressure under baseline conditions at ventricular volumes appropriate for dog. The cardiac cycle timing parameters $T_{M}$ and $T_{R}$ were set to the values used by Beard ${ }^{14}$. Heart rate parameters $H_{0}$ and $H_{1}$ were determined as described above. The baseline resistance and compliance parameters $R_{o u}, R_{A o}, R_{A 0}, R_{V}, C_{A 0}, C_{V 0}$, and $V_{V 01}$ were chosen so that under baseline conditions (Figure 5), the mean pressure is $100 \mathrm{mmHg}$, the diastolic and systolic pressures are 85 and $115 \mathrm{mmHg}$, and the ejection fraction is 0.58 . (The values for these seven parameters do not represent a unique set that gives these outputs under baseline conditions). The parameters $\gamma_{V}$ and $\tau_{c V}$ were set to match measurement of stress relaxation in the canine jugular ${ }^{15}$.

The remaining seven parameters in the cardiac and circulation model component $\left(\alpha_{1}, \alpha_{2}, \alpha_{3}, \alpha_{4}, F_{0}, F_{1}\right.$, and $\left.\tau_{A R}\right)$ were identified by simulating the responses of the system to volume infusion and hemorrhage, as detailed below in Results.

\section{Model Components 4: Autonomic system}

The whole-body sympathetic tone is represented in the model by the variable $\phi_{S N}(t) \in(0,1)$ and is determined by the baroreflex arc:

$$
\frac{d \phi_{S N}}{d t}=f_{S N}\left(1-\phi_{S N}\right)-f_{B R} \phi_{S N}
$$



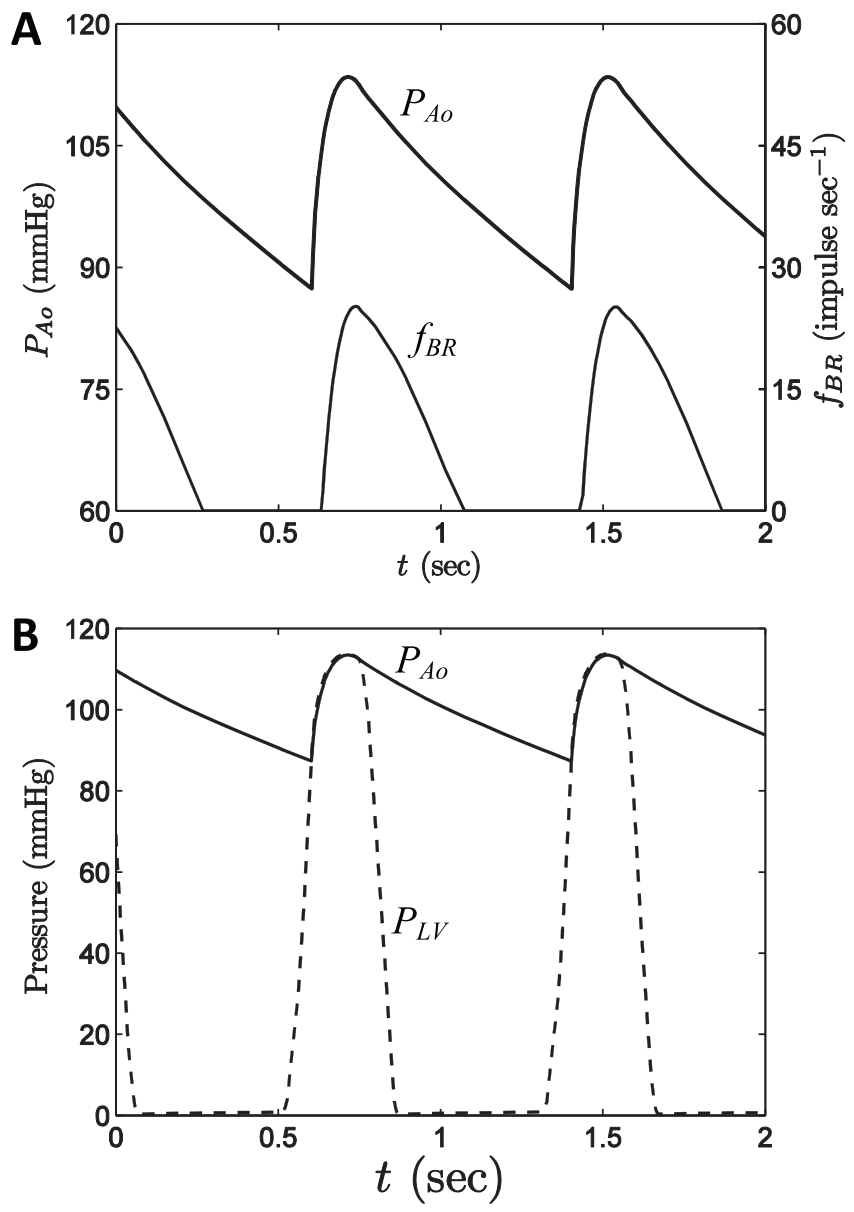

Figure 5. Baseline model operation. A. Model-predicted aortic pressure and baroreflex firing rate, obtained with $Q_{\text {in }}=0.5835 \mathrm{ml}$ $\mathrm{min}^{-1}$. This simulation represents a period steady-state of the model, in which $Q_{\text {in }}=Q_{\text {out }}$ and average pressure is $100 \mathrm{mmHg}$. B. Modelpredicted aortic and left-ventricular pressures are plotted for the baseline period steady state.

Thus, in the absence of baroreflex firing, the sympathetic tone will approach the maximum value of 1 . The constant parameter $f_{S N}$ is set so that under baseline conditions $\phi_{S N}(t)=0.25$. Thus, following a rapid severe drop in pressure $\phi_{S N}(t)$ will approach a value that is four times the baseline value.

\section{Model Components 5: Renin-angiotensin system}

The state of the renin-angiotensin system is captured by two variables: $\phi_{R}(t) \in(0,1)$ and $\phi_{A 2}(t) \in(0,1)$, which represents plasma renin and angiotensin II activity governed by the combined action of sympathetic tone and pressure on renin release.

The plasma renin variable $\phi_{R}(t)$ is governed by

$$
\begin{aligned}
& \tau_{R} \frac{d \phi_{R}}{d t}=\phi_{R}^{\infty}-\phi_{R} \\
& \tau_{P} \frac{d \bar{P}}{d t}=P_{A}-\bar{P} \\
& \phi_{R}^{\infty}=\frac{1}{2}\left(1-\tanh \left(\frac{\bar{P}-g \phi_{S N}-P_{1}}{P_{2}}\right)\right),
\end{aligned}
$$

where $\phi_{R}^{\infty}$ decreases with increasing time-averaged arterial pressure $\bar{P}$. This formulation assumes that the relationship between steadystate $\phi_{R}$ and pressure $\bar{P}$ is shifted by the sympathetic tone. Based on observations showing that plasma renin activity and angiotensin II levels are nearly perfectly linearly related in vivo ${ }^{16}$, we assumed that $\phi_{A 2}(t)$ follows $\phi_{R}(t)$ according to

$$
\tau_{A 2} \frac{d \phi_{A 2}}{d t}=\phi_{R}-\phi_{A 2}
$$

The five parameters invoked in this model component $\left(\tau_{R}, \tau_{A 2}, g, P_{1}\right.$, and $P_{2}$ ) were identified based on comparing simulations to measurements of pressure, heart rate, and plasma renin activity in rabbit during graded hemorrhage, as detailed below in Results. (The simulation parameter $\tau_{P}$ was arbitrarily set at 15 seconds).

\section{Model Components 6: Neurohumoral control of pressure- diuresis/natriuresis}

Regulation of body-fluid volume is assumed governed by a linear pressure-diuresis relationship:

$$
Q_{\text {urine }}= \begin{cases}0, & \bar{P}<P_{s} \\ \min \left(k_{1} \cdot\left(\bar{P}-P_{s}\right), 10 \mathrm{ml} \mathrm{min}^{-1}\right), & \bar{P} \geq P_{s}\end{cases}
$$

where $Q_{\text {urine }}$ is rate of volume output via the kidneys and $k_{1}$ is the slope of the relationship between $Q_{\text {urine }}$ and pressure. The model variable $P_{s}(t)$ is the variable offset of the pressure-diuresis relationship, which is controlled by sympathetic tone and angiotensin II level:

$$
\begin{aligned}
& P_{s}^{\infty}=P_{s, \text { min }}+\frac{P_{s, \text { max }}-P_{s, \min }}{2}\left(1+\tanh \left(\frac{\phi_{S N}+\phi_{A 2}-\phi_{0}}{\phi_{1}}\right)\right) \\
& \tau_{k} \frac{d P_{s}}{d t}=P_{s}^{\infty}-P_{s} .
\end{aligned}
$$

The constant parameters $P_{s, \text { min }}, P_{s, \max }, \phi_{0}$, and $\phi_{1}$ determine how the acute pressure-diuresis relationship shifts in response to changes in the tone variables $\phi_{S N}$ and $\phi_{A 2}$. Thus it is assumed that $\phi_{S N}$ and $\phi_{A 2}$ have equal and additive effects on renal function. Equation (22) assumes that changes in $P_{s}$ governed by sympathetic tone and the renin-angiotensin system occur with a time constant $\tau_{k}$. Furthermore, the maximal rate of urine production is set to $10 \mathrm{ml} \mathrm{min}^{-1}$.

The parameters $k_{1}, P_{s, \max }, P_{s, \min }, \phi_{0}$, and $\phi_{1}$, were set by matching model predictions to data on the pressure diuresis relationship under physiological conditions, with angiotensin II infusion, and with administration of an angiotensin converting enzyme (ACE) inhibitor. To compare predictions of Equation (21) and Equation (22) to renal output measurements under ACE inhibition, we assumed that for the chronic measurements, sympathetic tone was maintained at its baseline value and $\phi_{A 2}=0$. Fitting data from Hall et al. ${ }^{17}$, it was estimated that $P_{s}^{\infty}\left(\phi_{S N}=0.25, \phi_{A 2}=0\right)=75 \mathrm{mmHg}$. Similarly, with angiotensin II infusion, we assumed that sympathetic tone was maintained at its baseline value and $\phi_{A 2}=1$. The data of Hall et al. yield an estimate of $P_{s}^{\infty}\left(\phi_{S N}=0.25, \phi_{A 2}=1.0\right)=125 \mathrm{mmHg}$ for these conditions. Finally, under baseline conditions, with $\bar{P}=100 \mathrm{mmHg}$, urine output was estimated to be $Q_{\text {urine }}=0.5835 \mathrm{ml} \mathrm{min}^{-1}$, based on volume infusion experiments described in the Results. With 
$k_{1}=0.125 \mathrm{ml} \mathrm{sec}^{-1} \mathrm{mmHg}^{-1}$ (see Results), and using the baseline $\phi_{A 2}$ value of 0.1864 (which is determined from blood withdrawal experiments; see Results), it follows that $P_{s}^{\infty}\left(\phi_{S N}=0.25, \phi_{A 2}=0.1864\right)$ $=95.3 \mathrm{mmHg}$. These estimates of $P_{s}^{\infty}$ at three different values of $\phi_{S N}+\phi_{A 2}$ were used to estimate the values of $P_{s, \max }, P_{s, \min }, \phi_{0}$, and $\phi_{1}$.

The time constant $\tau_{k}$ was determined from data on the rate of urine production following infusion of blood leading to an acute increase in pressure and drop in $\phi_{S N}$ and $\phi_{A 2}$. Analysis of data from these experiments is detailed below in the Results together with a direct comparison between the data of Hall et al. ${ }^{17}$ and model simulations.

\section{Results}

Results 1: Response to volume infusion

Guyton and colleagues conducted experiments in which a large amount of blood was infused into an anesthetized dog, resulting in a rapid increase in total blood volume of $45 \%$ compared to initial baseline value. The model of Equation (1)-Equation (22) was simulated and compared to data from this experiment to identify adjustable model parameters and to probe the predicted response of unmeasured model variables to this protocol.

Figure 6 shows the predicted effects of infusing $45 \%$ of initial baseline blood volume in a normal animal on arterial pressure, cardiac output, rate of urine formation, blood volume, sympathetic tone, and renin-angiotensin level together with experimental data obtained from Dobbs et al..$^{18}$, Guyton et al. ${ }^{1}$, and Prather et al. ${ }^{19}$ on the first four variables. (For these experiments Guyton et al. reported a baseline mean arterial pressure of approximately $115 \mathrm{mmHg}$. Since all other data sets analyzed here have a baseline pressure of $100 \mathrm{mmHg}$, $15 \mathrm{mmHg}$ was subtracted from the data from these experiments to achieve consistency). The initial steady state of the model was obtained based on a constant infusion of $Q_{i n}=0.5835 \mathrm{ml} \mathrm{min}^{-1}$, which matches the mean reported rate of volume output for the baseline data. For times $0<t<5 \mathrm{~min}, Q_{\text {in }}$ was increased to $0.5835+126.82 \mathrm{ml} \mathrm{min}^{-1}$, to result in a total excess volume of $634 \mathrm{ml}$. After the five-minute infusion, $Q_{i n}$ was returned to the baseline value of $0.5835 \mathrm{ml} \mathrm{min}^{-1}$. The model is able to effectively reproduce the trends in the experimentally measured variables, and predicts that $\phi_{S N}$ and $\phi_{R A}$ drop to less than one half of their resting values following the volume infusion in response to the transient increase in pressure. These reductions in $\phi_{S N}$ and $\phi_{R}$ confer increases in vascular compliances helping to accommodate the substantial volume increase. As volume is removed from the system $\phi_{S N}$ and $\phi_{R}$ return toward the baseline values, with $\phi_{S N}$ responding substantially faster than $\phi_{R^{*}}$

The data plotted in Figure 6, along with data observations on graded hemorrhage, were used in identifying several adjustable parameters in the model. (See following section for details).

\section{Results 2: Response to hemorrhage}

Data from Quail et al. ${ }^{20}$ on heart rate, pressure, and plasma renin activity following graded blood withdrawal in rabbits were used to identify parameters associated with the cardiovascular mechanics and the renin-angiotensin system. Figure 7 shows data measured in normal rabbits, where the heart rate measurements have been scaled to a baseline value of 75 beats $\min ^{-1}$, renin activity is scaled to maximum value of 1 , and mean pressure is scaled to an initial baseline value of $100 \mathrm{mmHg}$. In the experiments, blood was withdrawn from the animals at a rate of $2 \%$ blood volume per minute. In model simulations, blood is withdrawn at the experimental rate starting at time 0 and with withdrawal stopped at time 17.5 minutes. For this experiment, the initial condition was identical to that used for the volume infusion experiments of Figure 6, and volume infusion and urine output were set to zero for the time course.

Model predictions of $H, \bar{P}$, and $\phi_{R}$ compare favorably to experimental data, with mean arterial pressure dropping from the initial value of $100 \mathrm{mmHg}$ to approximately $93 \mathrm{mmHg}$ at the end of the blood withdrawal. The observed increase in heart rate is associated with an increase in $\phi_{S N}$ from baseline level of 0.25 to 0.81 , and an increase of $\phi_{R}$ from baseline level of 0.186 to 0.668 , at the termination of the withdrawal period. During this period, arterial blood pressure is protected from a more severe reduction in large part by an increase in vascular tone mediated by increases in sympathetic tone and angiotensin II level. Simulated cardiac output (not shown) also drops during blood withdrawal, reaching a minimum value of $690 \mathrm{ml} \mathrm{min}^{-1}$ (approximately $46 \%$ of baseline flow) at 17.5 minutes.

The model is not able to capture the sharp, almost step-like, observed increase in renin that occurs following the 10-minute time point (corresponding to $20 \%$ of blood withdrawn). Specifically, the model predicts a more graded response, without the delay observed in the data. A better match to the data might be achieved by incorporating a delay into the governing equations, or by simulating the renin-angiotensin system using a higher-order system of differential equations than Equation (19) and Equation (20). The model predicts that $\phi_{S N}$ and $H$ peak at the end of the withdrawal period and partially recover toward baseline levels within a few minutes after the end of blood withdrawal. The renin-angiotensin tone is predicted to remain at an elevated level after the withdrawal. The partial drops in $\phi_{S N}$ and $H$ are associated with a graded decrease in pressure continuing over the entire simulated time course, with pressure reaching approximately $85 \mathrm{mmHg}$ at 30 minutes. Pressure continues to drop after the end of the withdrawal period due in part to the action of the whole-body autoregulatory mechanism governed by Equation (16) and Equation (17). Via this mechanism, the drop in flow associated with hemorrhage causes a reduction in arterial resistance, resulting in a recovery in flow and a continuing drop in pressure.

The model predictions in Figure 6 and Figure 7 are most sensitive to the values of 13 parameters from the model components of circulatory mechanics, the renin-angiotensin system, and the kinetics of pressure-diuresis: $\alpha_{1}, \alpha_{2}, \alpha_{3}, \alpha_{4}, F_{0}, F_{1}, \tau_{A R}, \tau_{R}, \tau_{A 2}, g, P_{1}, P_{2}$, and $t_{K}$. The values of these parameters were adjusted to match the data plotted in Figure 6 and Figure 7.

\section{Results 3: Chronic renal function curves}

The steady-state relationship between arterial pressure and rate of urine output is obtained from model simulations by varying $Q_{i n}$, the rate of volume infusion, and attaining steady-state model predictions of steady state pressure where $Q_{\text {in }}=Q_{\text {urine }}$. Figure 8A plots the predicted relationship between rate of urine output and mean arterial pressure under three different conditions: (1) the normal physiological state; (2) $\phi_{R}$ clamped at 0 , representing complete block of 

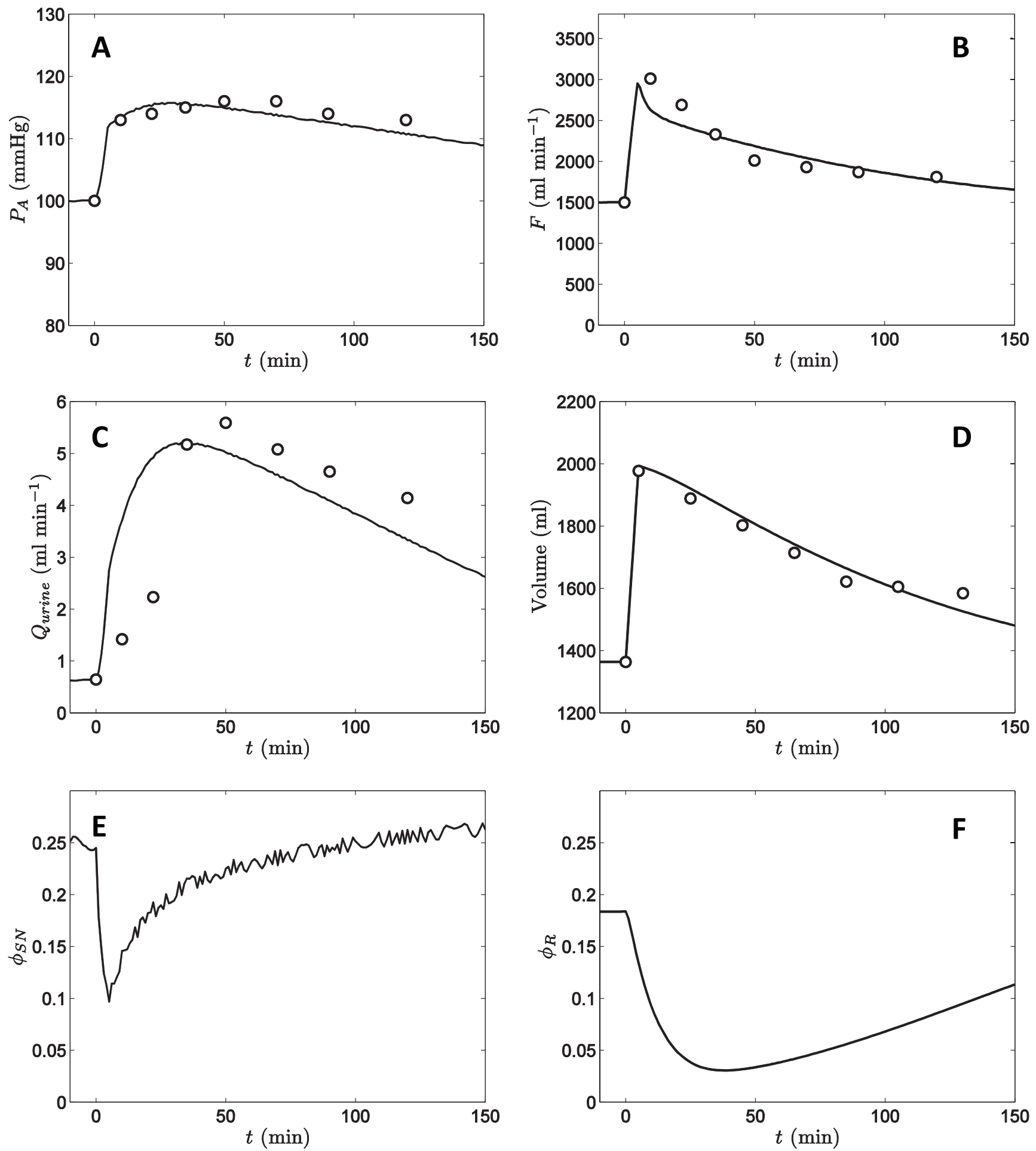

Figure 6. Simulation of arterial pressure, cardiac output, rate of urine formation, blood volume, $\phi_{S N}$, and $\phi_{R}$ following infusion of $45 \%$ of initial baseline blood volume in a normal animal. Data on arterial pressure, cardiac output, rate of urine formation, and blood volume are obtained from Dobbs et al..$^{18}$, Guyton et al. ${ }^{1}$, and Prather et al. ${ }^{19}$. The initial steady state of the model was obtained based on a constant

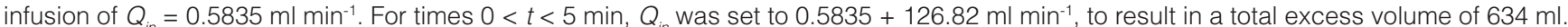
The initial condition for the simulation was obtained by setting $Q_{i n}=0.5835 \mathrm{ml} \mathrm{min}^{-1}$ and running the model to obtain the steady state. 

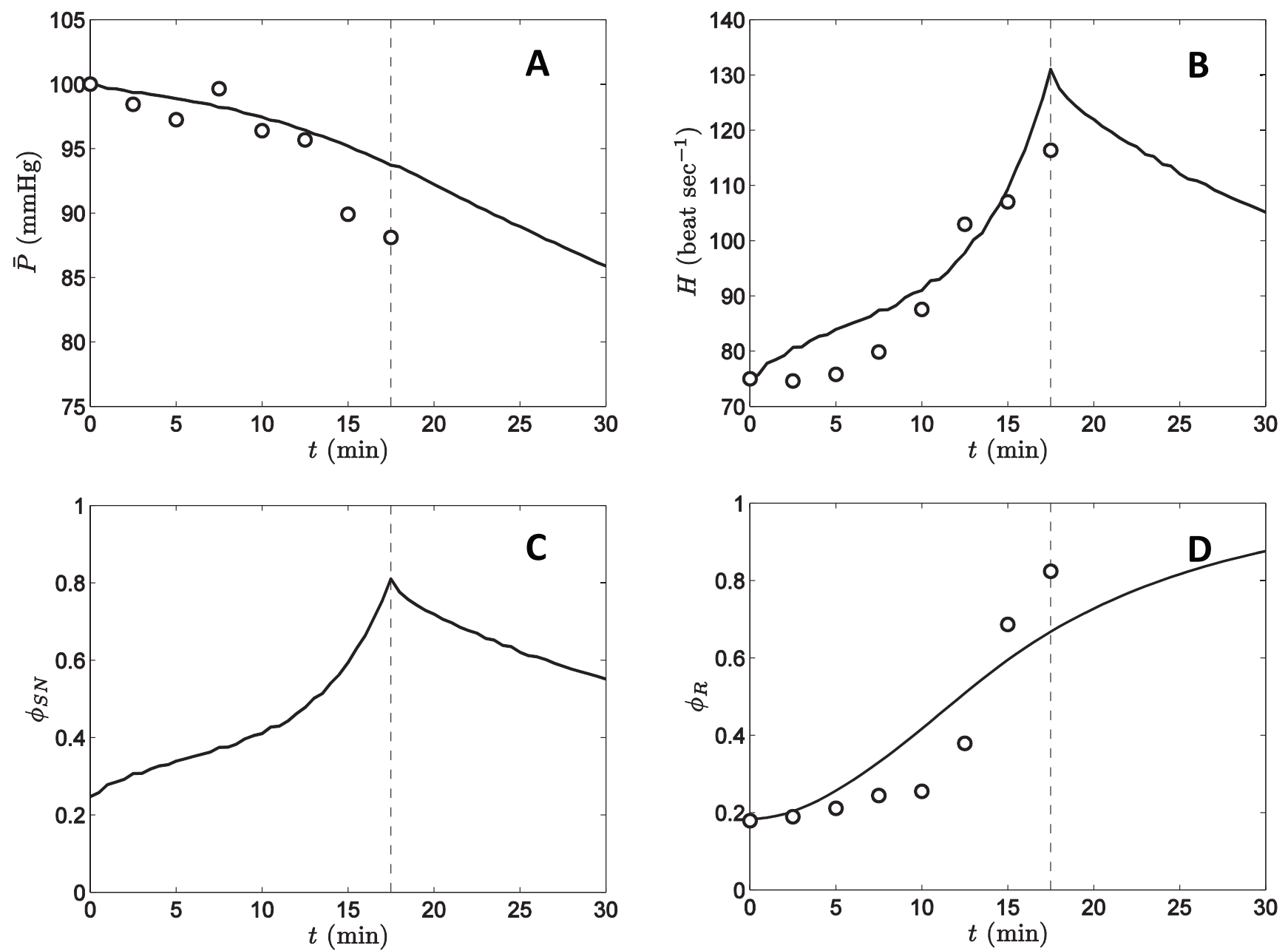

Figure 7. Simulation of system response to hemorrhage. The experimental and simulation protocol is to withdraw blood at a rate of $2 \%$ of initial total volume per minute, starting at time 0 . The end of the withdrawal period, 17.5 minutes, is indicated in by dashed line in all plots. Model simulations are compared to data for mean pressure $(\mathbf{A})$, heart rate $(\mathbf{B})$, and plasma renin activity $(\mathbf{D})$. Panel $\mathbf{C}$ plots the modelpredicted sympathetic tone during the protocol. The initial condition is the same baseline condition used for the simulations of Figure 6. Data from heart rate, pressure, and plasma renin activity following graded blood withdrawal are from Quail et al. ${ }^{18}$ with pressure and heart rate scaled as described in the text.

angiotensin converting enzyme (ACE); and (3) $\phi_{R}$ clamped at 1 , representing infusion of saturating levels of angiotensin II. Note that the pressure-diuresis relationship is plotted in the traditional manner with arterial pressure on the abscissa and rate of volume excretion on the ordinate, even though in these simulations $Q_{i n}$ is varied and steady-state $\bar{P}$ is computed as a function of $Q_{i n}$.

Model predictions are compared to data from Hall et al. ${ }^{17}$ on steady-state pressure at different levels of salt/volume loading in normal dogs, dogs infused with ACE inhibitor, and dogs infused with angiotensin II. Model simulations effectively match experimental data, validating the assignment of parameter values described above under "Neurohumoral Control of PressureDiuresis/Natriuresis".

Figure $8 \mathrm{~B}$ illustrates model-predicted steady-state renin and angiotensin II activities $\left(\phi_{R}\right.$ and $\left.\phi_{A 2}\right)$ and sympathetic tone $\left(\phi_{S N}\right)$ as functions of $Q_{i n}$ for the normal case. (Recall that from Equation (20) angiotensin II activity $\phi_{A 2}$ is equal to $\phi_{R}$ in the steady state). As $Q_{\text {in }}$ is increased $\phi_{R}$ and $\phi_{A 2}$ decrease to maintain pressure at nearly a constant level of the simulated range of volume/salt loading. The sympathetic tone on the other hand remains nearly constant around the baseline level of 0.25 . Sympathetic tone remains constant because in the model the only determinant of $\phi_{S N}$ is the baroreceptor afferent firing rate, which effectively adapts to the small changes in pressure that are associated with the simulated range of $Q_{i n}$. (Thus, the model does not capture suppression of sympathetic tone typically observed with chronic salt/volume loading).

\section{Results 4: Response to chronic baroreflex stimulation}

Numerous studies have demonstrated that chronic stimulation of the carotid baroreceptor afferent nerve with implantable devices results in a prolonged decrease in mean arterial pressure ${ }^{21,22}$. Figure 9 illustrates a representative data set from Lohmeier et al. ${ }^{23,24}$ for which 

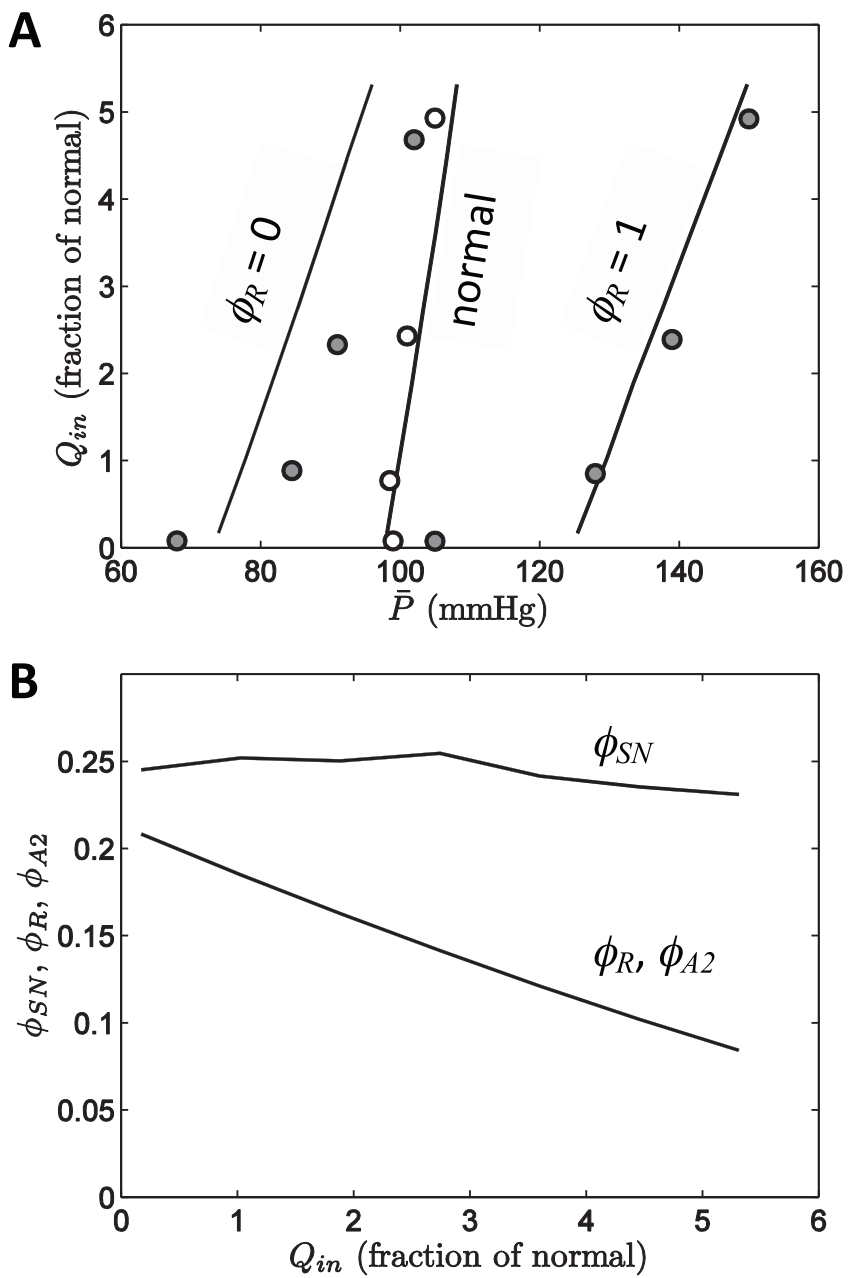

Figure 8. Renal function curves. A. Model predictions are compared to data on the steady-state relationship between mean arterial pressure and rate of volume infusion (equal to rate of urine output) for normal conditions, for angiotensin converting enzyme inhibition $\left(\phi_{R}=0\right)$, and for angiotensin II infusion $\left(\phi_{R}=1\right)$. Data are obtained from Hall et al. ${ }^{17}$, in which net salt output is reported under these three conditions. Rate of urine volume production is assumed proportional to rate of sodium excretion, and normalized to the rate of urine production at baseline conditions $(\bar{P}=100 \mathrm{mmHg})$ for the normal case. B. Model prediction for steady-state renin and angiotensin II activities $\left(\phi_{R}\right.$ and $\left.\phi_{A 2}\right)$ and sympathetic tone $\left(\phi_{S N}\right)$ are plotted as functions of $Q_{i n}$ for the normal case (without $\phi_{R}$ clamped).

baroreflex stimulation was applied in dogs for a seven day period, followed by several days of recovery. During the stimulation period, mean arterial pressure drops by approximately $20 \%$, renin activity by approximately $35 \%$, and sympathetic tone (experimentally assayed indirectly by plasma norepinephrine) drops by approximately $55 \%$.

To simulate this experiment, a constant was added to the modelpredicted baroreceptor firing rate in the equation for sympathetic tone. Thus Equation (18) was replaced by

$$
\frac{d \phi_{S N}}{d t}=f_{S N}\left(1-\phi_{S N}\right)-\left(f_{B R}+f_{s t i m}\right) \phi_{S N}
$$
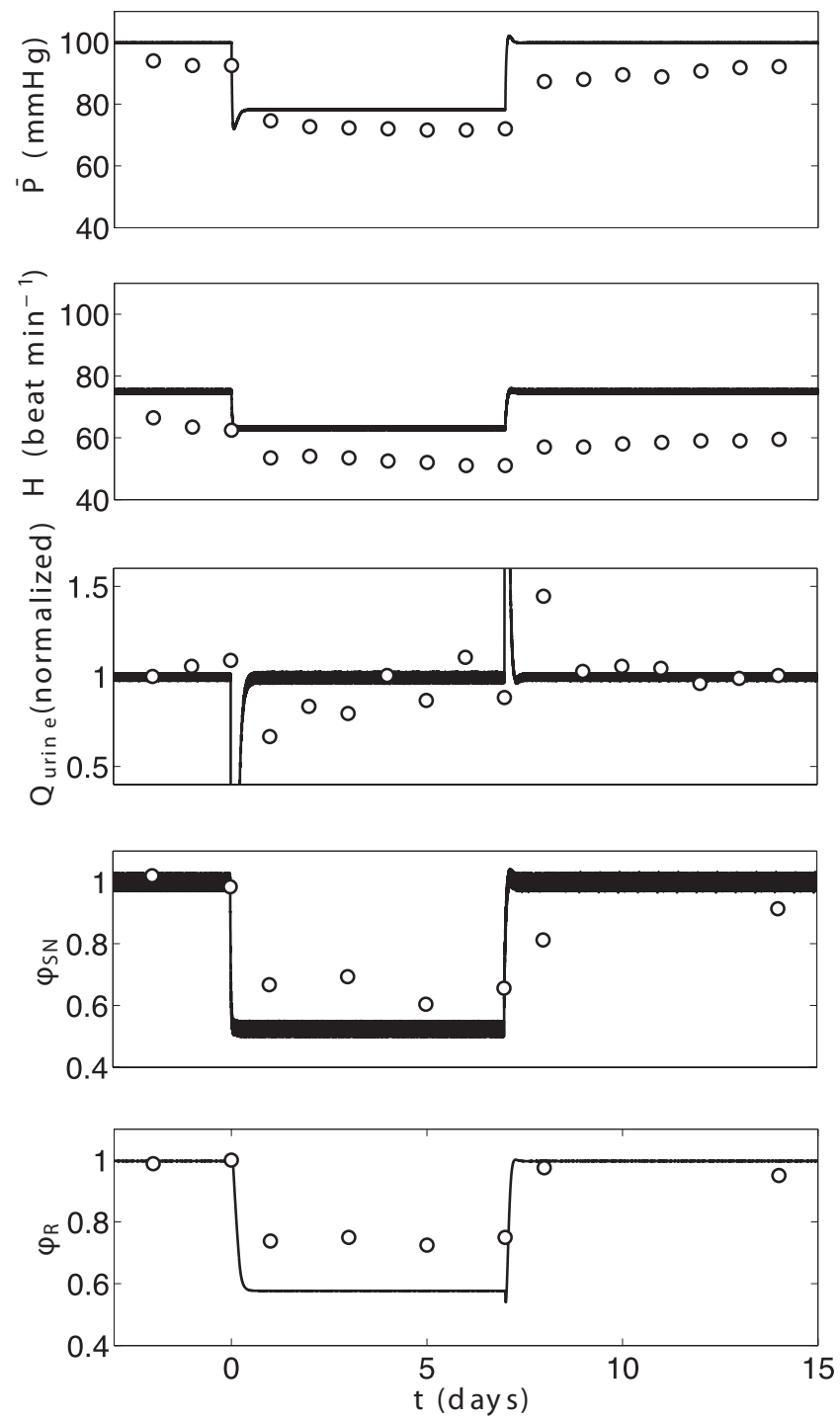

Figure 9. Response to chronic baroreflex stimulation. Electrical stimulation of the carotid baroreflex afferent nerve is simulated by modifying the normal model by replacing Equation (18) in the normal model with Equation (23) during the baroreflex stimulation period (for a 1-week period starting on day 0). Data on mean pressure, heart rate, urine output, plasma norepinephrine (plotted as $\phi_{S N}$ ), and plasma renin activity (plotted as $\phi_{R A}$ ) are obtained from Lohmeier et al. ${ }^{23,24}$.

where $f_{\text {stim }}$ is a parameter adjusted to match the experimental data. Using $f_{\text {stim }}=6.5 \mathrm{sec}^{-1}$ yields predictions that effectively match the observations of Lohmeier et al. ${ }^{23,24}$ (Figure 9). Indeed, given that only one parameter was adjusted, the comparison of model predictions to the five variables plotted in the figure represents a strong validation test for the model. In particular, the observed reduction in renin activity with baroreflex stimulation represents a phenomenon that has not been captured by previous modeling efforts ${ }^{22,24}$. Here, the phenomenon emerges as a property of the integrated computational model: stimulation of the baroreflex and associated drop in sympathetic tone is predicted to result in a drop in renin production even with the long-term drop in mean pressure. 
Results 5: Exploration of the etiology of primary hypertension

Pettersen et al. ${ }^{25}$ demonstrate that impairment of the baroreflex caused by stiffening of arterial vessels represents a viable hypothesis for the etiology of primary hypertension. Specifically, using a closed-loop cardiovascular mechanics model coupled to a detailed model of the baroreflex $\operatorname{arc}^{26}$, Pettersen et al. ${ }^{25}$ show that when the stiffness of the aorta is increased to represent changes in mechanical properties associated with ageing, the model based on their mechanogenic hypothesis predicts a substantial increase in mean arterial pressure with age. By assuming a constant blood volume for all age groups, the model explicitly did not account for the regulation of plasma volume and salt through the kidney and the renin-angiotensin system following from the hypothesized shift in the renal pressure-diuresis/natriuresis function curve. The rationale for not including adaptive mechanisms likely to partially ameliorate the effects of arterial stiffening on blood pressure was to test the explanatory sufficiency of the mechanogenic hypothesis by showing that it would predict a stronger relation between blood pressure increase and age than empirically observed. As a first step towards a complete merge of physiological renal function with the model developed by Pettersen et al. to expand the prediction space of the mechanogenic hypothesis, we studied how the current model responded to changes in the aortic compliance.

Using the current model, which does account for blood volume regulation by the kidneys, the hypothesis of Pettersen et al. may be further analyzed by determining how simulations of the current model respond to changes in the aortic compliance. Figure 10A (solid line) plots predicted steady-state mean arterial pressure as a function of relative aortic stiffness, $C_{A o}^{0} / C_{A o}$, where $C_{A o}^{0}$ is the baseline normal value of aortic compliance, and $C_{A o}$ is the value used to obtain the pressures reported in the Figure. The maximum simulated relative stiffness, $C_{A o}^{0} / C_{A o}=4$, is approximately the average relative stiffness for the 75 -year-old population simulated in Pettersen et al. ${ }^{25}$. This 4-fold increase in aortic stiffness increases the predicted mean pressure from 100 to $128 \mathrm{mmHg}$, with systolic/diastolic ratio increasing from $115 / 90$ to $139 / 117$. Furthermore, over the simulated range of stiffness model-predicted $\phi_{S N}$ increases from 0.25 to 0.45 and $\phi_{R}$ increases from 0.186 to 0.59 (Figure 10B) with a heart rate increase of approximately $25 \%$ as mean pressure increases from 100 to $128 \mathrm{mmHg}$.

As expected, this predicted pressure increase is less than that predicted for a model that does not account for blood volume regulation. (The model of Pettersen et al. predicts a mean arterial pressure 150 $\mathrm{mmHg}$ for 75 -year-old group). The difference between the predictions of the current model and that of Pettersen et al. is explained primarily by blood volume regulation: with a 4-fold increase in aortic stiffness the current model predicts the active blood volume to decrease from 1364 to $1050 \mathrm{ml}$ (Figure 10C). This result supports the view that normal functioning kidneys are able to partially compensate for age-related increases in arterial stiffness ${ }^{25}$.

Therefore this model demonstrates that it is possible to capture the age-related phenotype of primary hypertension as emerging solely from changes to the mechanical properties of the aorta and carotid arteries. This may seem surprising since the simulation results plotted in Figure 10 are obtained with mechanical properties of all components of the vasculature other than large vessels associated with
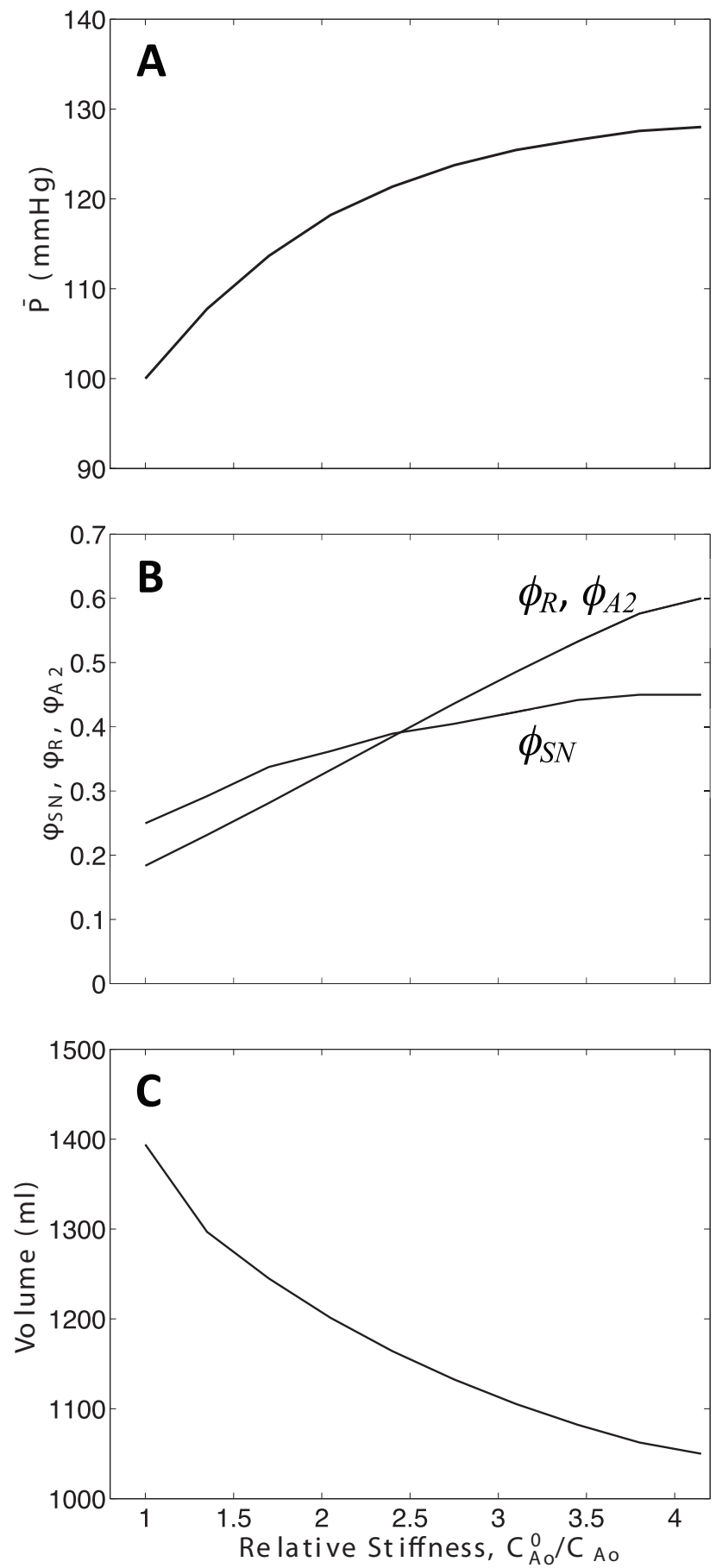

Figure 10. Effects of arterial stiffening on mean pressure. A. The model predicted for the steady-state mean arterial pressure is plotted as a function of relative aortic stiffness, $C_{A o}{ }^{0} / C_{A o}$, where $C_{A o}^{0}$ is the baseline normal value of aortic compliance, and $C_{A o}$ is the value used to obtain the simulated pressure. As stiffness is increased (as compliance is decreased), predicted mean pressure increases. Calculations assume normal salt/volume loading, $Q_{\text {in }}=0.5835 \mathrm{ml}$ $\mathrm{min}^{-1}$, resulting in a mean pressure of $100 \mathrm{mmHg}$ at $C_{A o}^{0} / C_{A o}=1$. B. Model-predicted steady-state sympathetic tone $\phi_{S N}$, plasma renin activity $\phi_{R}$, and angiotensin-II activity $\phi_{A 2}$ are plotted as functions of $C_{A o}{ }^{0} / C_{A o}$. C. Predicted blood volume is plotted as a function of $C_{A o}{ }^{0} / C_{A o}$ 
the baroreflex remaining normal. Indeed, it is expected that vascular stiffening would directly impact other physiological processes. For example, Beard and Mescam demonstrated that stiffening of renal arteries can explain the shift in the acute pressure-diuresis curve associated with changes in mean pressure in Dahl $\mathrm{S}$ rats ${ }^{27}$. For the model results illustrated in Figure 10 (representing normal kidney function), the predicted increase in mean pressure associated with increased vascular stiffness is partly due to an increase in the pressure that is required to elicit a given baroreceptor firing rate. As a result, the relationship between baroreceptor firing and mean arterial pressure is shifted to higher pressure in hypertension, as has been observed clinically and in animal models ${ }^{7}$. This phenomenon has been called "adaptation" or "resetting". Yet, even though model predictions are in agreement with the observed adaptation of the baroreflex, model predictions are not in agreement with the interpretation that, since baroreceptors adapt, "they cannot participate in the long-term control of arterial pressure" $"$. By contrast, the observed adaptation is a crucial component of the mechanogenic mechanism simulated here. Because stiffening causes an adaptation of the relationship between pressure and baroreceptor firing rate, vascular stiffening can cause a chronic increase in stable mean pressure. In other words, since baroreceptors adapt, they can contribute to the etiology of hypertension.

\section{Summary and conclusions}

A computational model (Source code is permanently available on: http://zenodo.org/record/7126 (10.5281/zenodo.7126)) was developed and identified to serve as a representation of the major physiological processes controlling arterial blood pressure. The design of the model was based on balancing the compromise between complexity/ physiological fidelity and identifiability, yielding a model of 16 state variables and 46 adjustable parameters. The model captures physiological phenomena occurring on times scales ranging from milliseconds (e.g. response of baroreceptor firing to arterial pressure) to days (e.g. response of mean pressure to chronic stimulation of baroreflex). It also effectively captures the response of the cardiovascular system to drastic perturbations, including infusion of $45 \%$ of blood volume, withdrawal of $35 \%$ of blood volume, electrical stimulation of the baroreflex, and the partial compensatory response of the kidneys to blood pressure increase accompanying arterial stiffening.

The major conclusions from this work are:

1. Over time frames ranging from seconds to weeks, arterial blood pressure is regulated primarily through the baroreflex arc, the reninangiotensin system, and the interoperation of the baroreflex arc and the renin-angiotensin system.

2. It is demonstrated how renal function is not the central or primary determinant of long-term arterial pressure in a model system that captures the observed behavior of the renin-angiotensin system, and chronic adaptation of the acute pressure-diuresis/natriuresis phenomena. This result contradicts the classical 'renocentric' view of the long-term control of blood pressure even though the model is built on the core assumption of the renocentric view that the pressure-diuresis relationship acts as a physiological input-output relationship in which pressure determines renal output. As a result, it is shown that hypertension does not necessarily indicate any degree of renal dysfunction.
3. The physiological response to chronic stimulation of the baroreflex can be explained by a mechanism in which arterial blood pressure is regulated primarily through the baroreflex arc and the interoperation of the baroreflex arc and the renin-angiotensin system.

4. Model simulations are consistent with the mechanogenic hypothesis $^{25}$ that arterial stiffening represents a contributing factor causing changes in pressure, sympathetic tone, and renal function associated with ageing. Through pressure-diuresis, normal kidney function is predicted to be able to partially ameliorate the effects of arterial stiffening on blood pressure.

Regarding the forth conclusion, model simulations predict that stiffening of the large arterial vessels that are associated with baroreceptors can contribute substantially to long-term changes in pressure. This result contradicts the 'renocentric' theory of blood pressure control even though the core assumption of the Guyton 'renocentric' theory of blood pressure control (that the pressure-diuresis relationship acts as a physiological input-output relationship in which pressure determines renal output) is a core assumption of this model. Although it has been argued that because its effective gain resets to a given mean arterial pressure the baroreflex cannot play an important role in long-term pressure regulation, this analysis shows how a dynamic system representing the baroreflex and capturing the observed resetting phenomenon can play a key role in the long-term control of pressure and the etiology of hypertension. Model simulations reveal that vascular stiffening increases sympathetic tone and shifts the effective baroreflex response to an increased pressure baseline. Because these changes cause an increase in angiotensin II activity and associated shift in the acute pressure-diuresis relationship, the renocentric ${ }^{7}$ and mechanogenic ${ }^{25}$ hypotheses for the etiology of primary hypertension could potentially be interpreted as compatible. However, as changes to renal function arise as downstream consequences of the mechanical remodeling, the mechanogenic explanation subsumes more of the biology involved in the etiology of hypertension compared with a renocentric explanation. This is a major conceptual advance that also provides a new interpretational framework for available experimental and clinical data.

The model developed here represents a simplification and several components are represented largely in phenomenological terms. A more detailed model may be constructed through integration of more biophysically based and detailed component models ${ }^{26-28}$ into the framework developed here. Regardless, the simplifications invoked in the model do not impact the major conclusions.

\section{Author contributions}

All authors contributed to model conception, development of the hypotheses to be tested, and design of computational experiments to analyze data and test hypotheses. DAB and SMB constructed the mathematical formulation of the model and wrote the computer code. DAB drafted the manuscript with all authors contributing to the content and/or revisions. 
Competing interests

The authors have no competing interests to disclose.

\section{Grant information}

This work was funded by the VPR Project, supported by NIH grant P50GM094503. KHP is supported by the Research Council of Norway under the eVITA program, project number 178901/V30.
The funders had no role in study design, data collection and analysis, decision to publish, or preparation of the manuscript.

\section{Acknowledgements}

The authors are grateful to Allen Cowley and David Mattson for valuable discussions.
1. Guyton AC: Circulatory physiology iii: Arterial pressure and hypertension Philadelphia, London, Toronto: W. B. Saunders Company; 1980. Reference Source

2. Montani JP, Van Vliet BN: Understanding the contribution of guyton's large circulatory model to long-term control of arterial pressure. Exp Physiol. 2009; 94(4): 382-388. PubMed Abstract | Publisher Full Text

3. Osborn JW, Averina VA, Fink GD: Current computational models do not reveal the importance of the nervous system in long-term control of arterial pressure. Exp Physiol. 2009; 94(4): 389-396. PubMed Abstract | Publisher Full Text | Free Full Text

4. Averina VA, Othmer HG, Fink GD, et al:: A new conceptual paradigm for the haemodynamics of salt-sensitive hypertension: A mathematical modelling approach. J Physiol. 2012; 590(Pt 23): 5975-5992.

PubMed Abstract | Publisher Full Text | Free Full Text

5. Hall JE, Mizelle HL, Hildebrandt DA, et al:: Abnormal pressure natriuresis A cause or a consequence of hypertension? Hypertension. 1990; 15(6 Pt 1): 547-559.

PubMed Abstract | Publisher Full Text

6. Guyton AC, Coleman TG, Granger HJ: Circulation: Overall regulation. Annu Rev Physiol. 1972; 34: 13-46.

PubMed Abstract | Publisher Full Text

7. Cowley AW Jr: Long-term control of arterial blood pressure. Physiol Rev. 1992; 72(1): 231-300. PubMed Abstract

8. Thomas SR, Baconnier P, Fontecave $\mathrm{J}$, et al: Saphir: A physiome core model of body fluid homeostasis and blood pressure regulation. Philos Trans A Math Phys Eng Sci. 2008; 366(1878): 3175-3197. PubMed Abstract | Publisher Full Text

9. Abram SR, Hodnett BL, Summers RL, et al.: Quantitative circulatory physiology: An integrative mathematical model of human physiology for medical education. Adv Physiol Educ. 2007; 31(2): 202-210. PubMed Abstract|Publisher Full Text

10. Beard DA: Tautology vs. Physiology in the etiology of hypertension. Physiology (Bethesda) 2013: 28(5): 270-271. PubMed Abstract | Publisher Full Text

11. Coleridge HM, Coleridge JC, Poore ER, et al:: Aortic wall properties and baroreceptor behaviour at normal arterial pressure and in acute hypertensive resetting in dogs. J Physiol. 1984; 350: 309-326. PubMed Abstract | Free Full Text

12. Sharpee TO, Miller KD, Stryker MP: On the importance of static nonlinearity in estimating spatiotemporal neural filters with natural stimuli. $J$ Neurophysiol. 2008; 99(5): 2496-2509.

PubMed Abstract | Publisher Full Text | Free Full Text

13. Chapleau MW, Lu J, Hajduczok G, et al:: Mechanism of baroreceptor adaptation in dogs: Attenuation of adaptation by the $\mathrm{k}+$ channel blocker 4-aminopyridine. J Physiol. 1993; 462: 291-306. PubMed Abstract | Free Full Text

14. Beard DA: Biosimulation: Simulation of living systems. Cambridge; New York: Cambridge University Press; 2012. Reference Source
15. Porciuncula $\mathrm{Cl}$, Armstrong GG Jr, Guyton AC, et al:: Delayed compliance in external jugular vein of the dog. Am J Physiol. 1964; 207: 728-732. PubMed Abstract

16. Cowley AW Jr, Guyton AC: Quantification of intermediate steps in the reninangiotensin-vasoconstrictor feedback loop in the dog. Circ Res. 1972; 30(5): $557-566$

PubMed Abstract | Publisher Full Text

17. Hall JE, Guyton AC, Smith MJ Jr, et al:: Blood pressure and renal function during chronic changes in sodium intake: Role of angiotensin. Am J Physiol. 1980; 239(3): F271-280. PubMed Abstract

18. Dobbs WA Jr, Prather JW, Guyton AC: Relative importance of nervous control of cardiac output and arterial pressure. Am J Cardiol. 1971; 27(5): 507-512. PubMed Abstract | Publisher Full Text

19. Prather JW, Taylor AE, Guyton AC: Effect of blood volume, mean circulatory pressure, and stress relaxation on cardiac output. Am J Physiol. 1969; 216(3): 467-472. PubMed Abstract

20. Quail AW, Woods RL, Korner PI: Cardiac and arterial baroreceptor influences in release of vasopressin and renin during hemorrhage. Am J Physiol. 1987; 252(6 Pt 2): H1120-1126. PubMed Abstract

21. Iliescu R, Irwin ED, Georgakopoulos D, et al:: Renal responses to chronic suppression of central sympathetic outflow. Hypertension. 2012; 60(3): 749-756. PubMed Abstract | Publisher Full Text | Free Full Text

22. Lohmeier TE, lliescu R: Lowering of blood pressure by chronic suppression of central sympathetic outflow: Insight from prolonged baroreflex activation. J Appl Physiol (1985). 2012; 113(10): 1652-1658. PubMed Abstract | Publisher Full Text | Free Full Text

23. Lohmeier TE, Irwin ED, Rossing MA, et al: Prolonged activation of the baroreflex produces sustained hypotension. Hypertension. 2004; 43(2): 306-311. PubMed Abstract | Publisher Full Text

24. Iliescu R, Lohmeier TE: Lowering of blood pressure during chronic suppression of central sympathetic outflow: Insight from computer simulations.

Clin Exp Pharmacol Physiol. 2010; 37(2): e24-33. PubMed Abstract | Publisher Full Text

25. Pettersen $\mathrm{KH}$, Bugenhagen SM, Nauman J, et al:: Arterial stiffening provides sufficient explanation for primary hypertension. 2013. Reference Source

26. Bugenhagen SM, Cowley AW Jr, Beard DA: Identifying physiological origins of baroreflex dysfunction in salt-sensitive hypertension in the dahl ss rat. Physiol Genomics. 2010; 42(1): 23-41. PubMed Abstract | Publisher Full Text | Free Full Text

27. Beard DA, Mescam M: Mechanisms of pressure-diuresis and pressurenatriuresis in dahl salt-resistant and dahl salt-sensitive rats. BMC physiology. 2012; $12: 6$.

PubMed Abstract | Publisher Full Text | Free Full Text

28. Beard DA, Neal ML, Tabesh-Saleki N, et al:: Multiscale modeling and data integration in the virtual physiological rat project. Ann Biomed Eng. 2012 40(11): 2365-2378

PubMed Abstract | Publisher Full Text | Free Full Text 


\title{
Open Peer Review
}

\section{Current Peer Review Status: ? $\checkmark$ ?}

\section{Version 1}

Reviewer Report 26 November 2013

https://doi.org/10.5256/f1000research.2510.r2540

(C) 2013 Hannaert P et al. This is an open access peer review report distributed under the terms of the Creative Commons Attribution License, which permits unrestricted use, distribution, and reproduction in any medium, provided the original work is properly cited.

\author{
Patrick Hannaert \\ INSERM U1082, Poitiers, France \\ François Guillaud \\ INSERM U1082, Poitiers, France
}

\section{SUMMARY}

This work by Beard et al., is an interesting, consequential and mind-tickling work, definitely worth considering and being published, provided that several issues are addressed.

\section{Strong points:}

Sound “horizontal" (multi-organ) physiological modelling \& simulation (following a systems/engineering analysis and conception). Modular, integrative, multi-dynamic approach.

Targeted, specific hypothesis - proposes one possible/sufficient etiogenic mechanism in the context of age-related hypertension.

Weak points:

Phenomenological equations.

Some conclusions drawn are too assertive (especially with respect to such a complex, multi-factorial, and evolving, pathology: hypertension); the likeliness of the conclusion appears strongly dependent on the limited physiology (and pathology) that is implemented.

\section{GENERAL}

In bio-medical research, it has become understood that mathematical modelling and simulation (MS) can provide a complementary approach to experiments and are a necessary response to extreme biological complexity. Indeed, biochemical and genetic circuits modelling are becoming mature and productive. On the other hand, there remains a very serious "modelling gap" to be 
filled between the ("low-level") biological/physiological processes and ("higher-level") pathophysiology.

Simply put, the "CV-circulatory-pressive" construct by Guyton and colleagues (started in the late 60's, with 1972 and 1992 milestones; Guyton et al., 1972; see Montani \& Van Vliet, 2009, Moss et al., 2012) remains a landmark, to the point that most, if not all modelling endeavours refer to it in one way or another. This is because it was an exhaustive attempt to describe the CV circulatory system in its physiological entirety, including high-level interactions such as an integrated CV and renal system.

Many versions, declinations (and improvements) do exist, including the late HumMod (Hester et al, 2011 should perhaps be cited, instead of Abran et al., 2007, since it is the current evolution of $\mathrm{QCP} / \mathrm{QHP}$ ). However, as mentioned by the Authors, for various reasons it is difficult or impossible to rationally understand and exploit those models (e.g. "too large" in the case of HumMod, or "not identified" as in the case of Guyton's models of 1972 or 1992).

Beard et al. propose here a "top-down", modular, "engineering" high-quality MS work, as a rationally exploitable (numerically identifiable) alternative to Guyton's model, in order to explore hypertension etiogenic hypothesis related to arterial stiffness, baroreflex and the reninangiotensin system (RAS). The main incentive relates to the controversed Guytonian view that long-term regulation of arterial pressure is driven by the kidney. In that context the Authors try to demonstrate that arterial stiffening (as in ageing) and/or the central nervous system (here simplified as the "baroreflex") could provide a sufficient explanation(s) of hypertension, through interactions with the kidney pressure-natriuresis function and the RAS.

The significance and relevance of this work for CV regulations and hypertension is obvious, although

(i) it is limited by the model itself (i.e. effectively implemented features, simplifications, phenomenological approach for equations, etc.) and

(ii) some of the conclusions/assertions should be tempered (among other reasons: given the multifactorial/multi-dynamic nature of $\mathrm{HT}$, that it has been resistant to investigation for more than a century now, and given the simplicity of the proposed construct).

\section{MAJOR COMMENTS}

In the targeted domain of the pathophysiology of blood pressure regulation and primary hypertension, this work proposes a rigorously constructed and parameterized mathematical model of the "long-term control of arterial pressure" (but see below). The authors should emphasize however that the model numerical instantiation centers on dog (or rabbit). Also of important note (this should be addressed in the revised version), no mention is made of the parameterization procedure and tools or sensitivity analysis.

Moreover, the parameterization is done under the (quite frequent) implicit hypothesis that arterial pressure regulation and other CV-related processes only differ quantitatively (e.g. in parameter values) and are otherwise qualitatively similar e.g. in dog, rabbit and man. This very point is rarely, if ever, addressed. We do feel that in the present turmoil of "physiological" models targeting 
*human* pathology, this should be, at minimum, mentioned and preferably justified. Given that the Authors target human hypertension, and especially given the phenomenological approach chosen, why did they not directly parameterize their model as a "human" CVR model?

As a matter of fact, the anatomy and mechanical/physiological/biochemical properties and functions of the human CV system, arteries, heart, kidney and RAS are, to the best of our knowledge, now understood and numerically characterized enough to allow for such a human quantitative approach to be adopted (especially with this articles chosen phenomenological "input-output" approach). This would have given some more resonance and relevance to the work. In particular, even if all CV-pressive, renal and hormonal processes proved to be the same between all these species, the possibility would remain that their relative contributions (and dynamics) to arterial pressure (dys-) regulations are different.

As their objectives, the Authors aimed to:

1. develop a "computer model capturing chronic adaptation of acute renal function in blood pressure control" as a more rigorous alternative to Guyton's circulatory model (Guyton et al., 1972) and to Guyton's-derived approaches, particularly SAPHIR (Thomas et al., 2008, Hernandez et al., 2011, could be cited too here, as well as Moss et al., 2012)

2. thus "capturing the relevant mechanisms".

These are very strong and ambitious objectives, and possibly ab initio flawed by the phenomenological (but otherwise legitimate) approach chosen.

Further, that this is "a model capturing chronic adaptation of acute renal function in blood pressure" cannot be claimed with such a simple kidney function, for example: the pressure-natriuresis ignoring actions of the ANS (autonomic nervous system) upon renal hemodynamics and tubular function and the angiotensin action on glomerular and tubular function (for instance Glomerular Tubulo Balance and $\mathrm{Na}$ /water reabsorption). Kidney nerve afferents are also ignored.

What is really addressed in this work are the interactions (possibly etiogenic) between the physiological (input/output) modules that are implemented.

Finally, after more than a century of physiological and clinical research, the etiology of primary hypertension remains unresolved; although it is established that (numerous), genetic, environmental, age-related and adaptive factors do intervene. Moreover, when in the realm of age-related hypertension the Authors do select putative etiogenic processes (arterial stiffening, reduced $B R$ receptors sensitivity) and explore potential interactions with RAS and kidney function (phenomenological pressure-natriuresis) in a simplified CV construct.

Driven by the objective (age related HT/arterial stiffening), the proposed model features six central physiological functionalities, as modules (model components) implementing:

MC1. "Aorta-large artery mechanics"

MC2. "Kinetics of baroreflex afferent firing"

MC3. "Mechanics of the heart and circulation"

MC4. "Autonomic system"

MC5. "Neuro humoral control of pressure diuresis/natriuresis"

MC6. "Renin-angiotensin system" 
For each one of these model components (physiological organ/process), the Authors should give, where possible, at least one published "first-principle" (physiologically-based) model as a reference, e.g. amongst others (i) Moss and Thomas, 2013 for the kidney/pressure-natriuresis under hormonal influence, (ii) Guillaud and Hannaert, 2010 and/or Claassen et al., 2013 for the RAS.

The model is first validated (short-term) vs haemorrhage and then volume expansion. Of note, no real validation criterion is given (except for visual adequation through the figures).

Secondly, the model is validated (long-term = days to weeks) using published steady-state pressure-natriuresis kidney function data. Although the Authors target long-term processes, 2 weeks (maximal simulated time here) is far too insufficient to refer to the CV, renal and central physiological mechanisms truly involved (months-to-years in dogs, years and decades in man).

\section{OTHER COMMENTS}

Is one of the input arrows missing in the model figure (fig 4)?

A model diagram (rather than the electrical analog presented), as well as for the main hypothesis (vs alternative(s)) would be welcome.

To what extent does this work differ from the previous work by the same group (cited, see

Pettersen et al., 2013)?

The Matlab(C) model code and scripts proposed (

http://zenodo.org/record/7126\#.UpOLNOJ21 pg) have been tested and verified by Dr F. Guillaud (ModTeam/Inserm U1082). The scripts could be run, the model could be simulated and results that were visually identical to those in the paper were obtained.

Competing Interests: No competing interests were disclosed.

We confirm that we have read this submission and believe that we have an appropriate level of expertise to confirm that it is of an acceptable scientific standard, however we have significant reservations, as outlined above.

Author Response 26 Nov 2013

.., ., USA

The reviewers have provided an extensive and insightful commentary on our paper. We agree with most of the points raised. We have extracted specific criticisms and responded to them below.

Weak points: Phenomenological equations;

This point is addressed in some detail in the responses to the first two reviewers, as well in response to specific points below.

Many versions, declinations (and improvements) do exist, including the late HumMod ( Hester et al, 2011 should perhaps be cited, instead of Abran et al., 2007, since it is the current evolution of QCP/QHP). 
The more recent citation has been inserted into the revised text.

...some of the conclusions/assertions should be tempered...

The validity of our conclusions is addressed extensively in our response to the first set of reviewers. In brief, we agree that some statements needed to be clarified and rephrased. See responses above for details.

The authors should emphasize however that the model numerical instantiation centers on dog (or rabbit); the parameterization is done under the (quite frequent) implicit hypothesis that arterial pressure regulation and other CV-related processes only differ quantitatively (e.g. in parameter values) and are otherwise qualitatively similar e.g. in dog, rabbit and man.

This is an important point that cuts across all areas of computational physiology. Largescale models are built based on data from multiple sources, multiple species, different conditions (in vitro, in vivo, etc.) In the end, all of our models are chimeras. We have taken the liberty of adapting the reviewers' language at the end of the discussion section of the revised paper. (We do point out that our implicit assumption is the central assumption of all biomedical research using animal models. It might not be a perfect assumption, but it does give us a starting point.)

Also of important note... no mention is made of the parameterization procedure and tools or sensitivity analysis.

Please see our detailed response to this point in the response to the first review.

For each one of these model components (physiological organ/process), the Authors should give, where possible, at least one published "first-principle" (physiologicallybased) model as a reference

This is an excellent idea in principle. However, it is not clear if it is possible. For example, we are unclear as to how the Moss and Thomas paper could be characterized as representing the hormonal influence on pressure-natriuresis from first principles. Specifically, the Moss and Thomas paper represents the action of angiotensin-II "via the parameter FrANG, which adjusts the response of the pressure-natriuresis mechanism." The parameter FrANG appears in the model equations as a factor multiplying a phenomenological expression for the fractional reabsorption, and the effect of the hormone is simulated by varying FrANG between "a maximal value of 1 (minimal angiotensin II) and a minimal value of 1/6 (maximal angiotensin II)". Thus, the model is every bit as "phenomenological" as the one presented here. (This point is not to be taken as a criticism of the excellent study of Moss and Thomas.)

The papers by Guillaud and Hannaert, and Claassen et al. do account for the RAS system with more physiological fidelity/detail than our model. We do disagree with the assertion that any of the models discussed here are built from "first" principles. All of these papers invoke the same sort of phenomenological expressions for certain components. 


\section{Although the Authors target long-term processes, 2 weeks (maximal simulated time here) is far too insufficient to refer to the CV, renal and central physiological mechanisms truly involved...}

The reviewers may be correct that our model is far too insufficient to provide insight into a pathophysiological mechanism that emerges over decades. Yet, we refer the reviewers (and the readers) to the stated conclusions in the paper.

\section{Is one of the input arrows missing in the model figure (fig 4)?}

The net volume output rate is Q_urine - Q_in. The figure has been revised to clarify this.

A model diagram (rather than the electrical analog presented), as well as for the main hypothesis (vs alternative(s)) would be welcome.

This has been added. (See Figure 4B.) The main hypothesis here is that pressure-diuresis acts as a physiological input-out relationship, and that renal function is controlled through the RAS and the autonomic system. Thus, fundamentally, the model is constructed to capture the Guyton concept of the long-term control of arterial pressure. (Therefore, it is not clear what alternatives we might indicate in a model diagram.)

\section{To what extent does this work differ from the previous work by the same group..?}

Please see the Section "Results 5: Exploration of the etiology of primary hypertension" in the revised text.

Competing Interests: No competing interests were disclosed.

Reviewer Report 14 November 2013

https://doi.org/10.5256/f1000research.2510.r2446

(C) 2013 Ottesen J. This is an open access peer review report distributed under the terms of the Creative Commons Attribution License, which permits unrestricted use, distribution, and reproduction in any medium, provided the original work is properly cited.

\section{Johnny Ottesen}

Department of Science, Systems, and Models, Roskilde University, Roskilde County, Denmark

Complex physiologically systems are often subdivided into parts and studied separately by experimental methods. But in experimental physiology, subsystems are not easily put back together and conclusions made concerning subsystems have limited validity for the original undivided system as their function and dynamics may have changed when isolated from one another. Parts that cannot be isolated experimentally may be studied (separately) using modeling. 
Mathematical modeling is able to unfold the influence that each of the processes has on the overall dynamic and thus modeling is highly adept at separating systems into components simple enough for their structures and functions to be studied in isolation. Mathematical modeling is the only controlled way to put the pieces back together by using equations that represent the system's components and processes, as well as its structures and interactions.

In "A computational analysis of the long-term regulation of arterial pressure" the authors follow this strategy nicely in a successful attempt to investigate what they denote the 'renocentric' theory by Guyton. Here the authors combine cardiovascular and baroreflex models with models of the renin-angiotensin system whereby some important conclusions are reached. Among these conclusions the authors emphasize that the baroreflex may play a key role in the long-term regulation of the arterial pressure and especially in hypertension in contrast to the Guytonian view.

The authors follow the physiologically structure well in their models as they carefully validate the different sub-models/elements by comparing them with existing various animal experiments. These sub-models are then integrated into a well-behaved and solid overall model.

However, there are some methodic drawbacks I would like to emphasize: Most model equations are in some sense ad-hoc choices (or phenomenological, as the authors mention at the very end). These equations are not based on first principles and hence they do not represent the underlying mechanisms. Instead the approach is to assume a first order transient toward some static sigmoidal relationship. In addition, the authors make a lot of self-references, which shadows the fact that many of the used sub-models are adjustment taken from elsewhere and ideas from other sources. Strengthening the paper with such information would definitely support the approach taken.

The paper is very well structured and written but a few misprints have sneaked in:

Formula (3): Should the fraction be / $C_{\mathrm{Ao}}$ instead of $C_{\mathrm{Ao}} /$ ? Formula (4): An index 0 is missing on $\mathrm{V}_{\mathrm{SA}}$ On page 4, coulomb 2, line 2: should be On page 4, coulomb 2, line 5: '... the population transition from an active state at a rate ...' Please state reference (or state that this is a hypothetical assumption)!

Competing Interests: No competing interests were disclosed.

\section{I confirm that I have read this submission and believe that I have an appropriate level of expertise to confirm that it is of an acceptable scientific standard.}

Author Response 26 Nov 2013

.., ., USA

Thank you for the attention to our paper and the constructive criticisms.

Most model equations are in some sense ad-hoc choices (or phenomenological, as the authors mention at the very end). These equations are not based on first principles 
and hence they do not represent the underlying mechanisms... In addition, the authors make a lot of self-references...

We agree with the reviewer's assessment. To reiterate a statement from the paper summary, the model represents a balance complexity/physiological fidelity and identifiability. The sensitivity analysis added to the revised paper indirectly addresses the issue of the phenomenological nature of model components. Finally, we have revised the text to clearly state the nature of the model earlier than the last paragraph of the paper. See third paragraph on page two, section on "Model Components 5", and first paragraph of the summary.

Also, we point out that while we have cited some previous modelling works, the majority of components used here have been constructed de novo, with the overall goal in mind. The varying elastance heart model is adapted from the cited reference. And of course there is nothing new about lumped-parameter models of the circulation. But most of the other (phenomenological) formulae are constructed to match the observed dynamics of responses of these systems to the experiments used to identify the model.

\section{Formula (3): Should the fraction be / $C_{\mathrm{Ao}}$ instead of $C_{\mathrm{Ao}} /$ ?}

No, it is correct in the paper. One way to see this is to think about the limit where $C_{\text {Ao }}$ goes to infinity. In this case the vessel becomes infinitely compliant if we wait long enough and thus does not support any finite pressure.

\section{Formula (4): An index 0 is missing on $V_{s A}$}

Fixed.

\section{On page 4, column 2, line 2: should be $d_{0}$}

Fixed

On page 4, column 2 , line $5:$ '... the population transition from an active state at a rate ...' Please state reference (or state that this is a hypothetical assumption)!

The assumption of Equation (7) is stated: "It is assumed that the baroreceptors within the population transition from an active to inactive state at a rate proportional to the firing rate". We do not know what factors are responsible for the slow "resetting" of the baroreflex response. But, it is compelling that this simple equation is able to capture the nonlinear phenomena of Figures 2 and 3.

Competing Interests: No competing interests were disclosed. 
(C) 2013 Osborn J et al. This is an open access peer review report distributed under the terms of the Creative Commons Attribution License, which permits unrestricted use, distribution, and reproduction in any medium, provided the original work is properly cited.

\section{John Osborn}

Department of Integrative Biology and Physiology, University of Minnesota, Minneapolis, MN, USA Viktoria Averina

University of Minnesota, Minneapolis, MN, USA

This paper by Beard et al makes three major claims: 1) to provide a mathematical description for Guyton's existing model; 2 ) to show how stimulation of the baroreflex arc can explain long-term changes in blood pressure via the renal function curve employed in Guyton's model; and 3) to show how arterial stiffening can lead to hypertension without renal injury. I commend the authors for making a much needed mathematical contribution to the field of computational physiology, where one of the most widely recognized and used models of long-term control of arterial pressure has only very rudimentary mathematical description and analysis published. Moreover, the authors are bridging the gap between the renocentric view of hypertension etiology and the emerging experimental data that points to an independent long-term role of neural regulation. For these reasons the paper is a valuable addition to the existing body of knowledge.

There are several issues however, that need to be addressed in a revised version of the paper. The overarching recommendation is to correct the paper's focus and claims to make them more consistent with the model design, as well as to make the model's description easier to understand. In particular, I believe the model should not be presented as a generic model with the "representation of the major physiological processes controlling arterial blood pressure" but instead be positioned as a conceptual framework which aims to illustrate how the baroreflex arc may be involved in long-term blood pressure control. Phenomenological mathematical representation, a small amount of detail, limited analysis, and quality of shown simulations are appropriate for a conceptual model but are insufficient for a model from which one could be extracting conclusions on the particular physiological mechanisms to be implicated in hypertension development.

\section{Suggested revisions:}

There are several versions of Guyton's model ranging from very simplistic to very complex ${ }^{1}$. Some models are extremely detailed but have a closed architecture (e.g. hummod.org). The model created by the authors is not sophisticated enough to correspond to most of these versions. Please state clearly which version of Guyton's model is being considered here. In my opinion, the model proposed here seems of approximately the same level of complexity to the one presented by Guyton and Coleman 'Long-term regulation of the circulation: interrelationships with body fluid volumes' in the text Physical bases of circulatory transport: Regulation and exchange. (1967) ${ }^{2}$

1. Focus the goals according to what was accomplished in the paper:

"capturing the relevant mechanisms in an identifiable mathematical model" is too overreaching since the three key equations $(18,19$, and 21$)$ do not capture mechanisms but are of phenomenological nature.

"probing hypotheses regarding the long-term control of arterial pressure" - the model is both too limited and phenomenological to allow for any testing of various hypotheses. It is, however, fit for providing a possible explanation for two 
hypotheses: roles of the baroreflex involvement and arterial stiffening. Otherwise, for testing, one would require a more mechanistic description, inclusion of other key organs and regulatory mechanisms, and a more rigorous mathematical analyses.

2. The authors provided an explanation of how the baroreflex may be involved in long-term control of blood pressure. This is a novel contribution to the field and should be the focus of the discussion:

- Add the discussion and references of experimental knowledge of baroreflex stimulation, inhibition, and especially long-term resetting.

Describe how Guyton's models incorporate neural control and explain the above finding, especially the effects of chronic stimulation and how it is different from the proposed model (e.g. ${ }^{3}$ )

Discuss existing knowledge or a possible physiological explanation for the finding that renin activity will be diminished with baroreflex stimulation.

- Discuss what other important physiological mechanisms are not reflected in the model and how they may play a role. In particular, explain why macula densa sodium delivery is thought not to be important enough to include in the model, despite the fact that it is known to be one of the key regulators of renin activity.

Do not confuse the model's predictions with its assumptions when discussing simulation results. The model assumes and is explicitly designed to tie the baroreflex, renin, and SNA with long-term pressure control. An explicitly designed assumption is not the same as prediction. Thus, statements like Conclusion 1 in "Summary and Conclusions" are inappropriate. The fact that the model's assumptions allow for physiologically plausible simulations merely supports the notion that baroreflex may be important in the way the authors have described it.

3. Improve the clarity of the mathematical description:

Provide motivation for the chosen elements of the model and outline the model in its entirety first. For example, a flowchart or a diagram of the model may help readers to grasp the overall idea.

Add at least a basic analysis of parameter sensitivity, with clear description in the Methods section.

In Table 1 please provide physiologically-based description of parameters rather than referring to the equations where they are used (e.g. Emax is the maximal leftventricular elastance, rather than "Varying elastance heart model, Equation (10)"). Otherwise, the definitions are circular: parameters are used in the equations without an explanation of their meaning in either the text or the table.

\section{References}

1. Guyton AC, Coleman TG, Granger HJ: Circulation: overall regulation.Annual Review of Physiology. 1972; 34: 13-44 PubMed Abstract | Publisher Full Text

2. Guyton AC, Coleman TG, Granger: Lowering of blood pressure during chronic suppression of central sympathetic outflow: insight from computer simulations. Physical bases of circulatory transport: Regulation and exchange. 1976.

3. Illiescu R, Lohmeier TE: Lowering of blood pressure during chronic suppression of central sympathetic outflow: insight from computer simulations.Clinical and Experimental Pharmacology and Physiology. 2010; 37 (2). PubMed Abstract | Publisher Full Text 
Competing Interests: No competing interests were disclosed.

\begin{abstract}
We confirm that we have read this submission and believe that we have an appropriate level of expertise to confirm that it is of an acceptable scientific standard, however we have significant reservations, as outlined above.
\end{abstract}

Author Response 26 Nov 2013

$\ldots$, , USA

We are grateful to the reviewers for paying such serious attention to our paper and offering many constructive criticisms. While we have revised the paper to address the concerns and clarify the presentation, we disagree with the general interpretation of our model as " $a$ conceptual framework which aims to illustrate how the baroreflex arc may be involved in longterm blood pressure control." As stated in the summary, the model "was developed and identified to serve as a representation of the major physiological processes controlling arterial blood pressure." For example, there is no equation or parameter value artificially injected into the model to require pressure-diuresis to not be able to return the pressure to a baseline value during baroreflex stimulation. In fact, we could parameterize the model so that the chronic pressure-diuresis phenomenon acts as an "infinite-gain" control system, always returning pressure to its set point. But in doing so, the model would not be able to match the data used to identify it, or to predict the data used to validate it.

Regarding the level of detail captured by the model, the reviewers are correct that at some level, all components of the model are phenomenological in nature. (This is true of all mathematical models.) Yet regarding the question around the level of detail and complexity of the model, we stand by the assertion that this model represents the most detailed model of long-term control of arterial pressure that is identified in the literature. (Here we are invoking the word "identified" to indicate that the model equations and associated parameter values are reported along with comparisons of model simulations of data used to identify the parameters.)

In fact, one of the unstated goals of this paper is to inject some clarity and rigor into this field with precise and concrete language. Therefore we do not know how to respond to the statement that our model "is not sophisticated enough to correspond to most of" the versions of the Guyton model because it is not clear, precisely, what is meant by "sophisticated" or "correspond". In discussing the Guyton models, we clearly state that: "The 1972 realization of the model invokes approximately 160 variables, and several hundred adjustable parameters. More recent versions of the model involve many thousands of variables and tens of thousands of parameters." Although it is true that our model has less variables and parameters than any version of the Guyton model that we are aware of, is the sophistication of a model measured purely by the number of variables or adjustable parameters? Since no version of the Guyton model captures pulsatile mechanics, no version of the model can capture the baroreceptor resetting phenomena illustrated in Figures 2 and 3 of our paper. Nor does the HumMod model capture the system response to baroreflex stimulation illustrated in Figure 9. Therefore one could argue that our 16-variable model is more sophisticated than the 5,000-variable Guyton model. 
Regarding the specific suggestions:

"capturing the relevant mechanisms in an identifiable mathematical model" is too over-reaching since the three key equations $(18,19$, and 21$)$ do not capture mechanisms but are of phenomenological nature.

More specifically, we stated that one of the "goal" of the study is "capturing the relevant mechanisms in an identifiable mathematical model." We believe we have done that. However, in the revised paper we have changed the goal to "representing the relevant mechanisms in an identifiable mathematical model."

"probing hypotheses regarding the long-term control of arterial pressure" - the model is both too limited and phenomenological to allow for any testing of various hypotheses.

Again, we counter that we have met the stated goal. We are not claiming to have proved any hypothesis. Yet we have introduced some important disproofs, as outlined in some of our major conclusions. Specifically, it is stated in the literature as 'scientific facts' that; the renal function curve represents the dominant long-term controller of blood pressure and, that since the baroreflex "resets" it cannot play a role in chronic changes in mean pressure. Our model analysis has cast significant doubt upon the validity of both of these hypotheses. Admittedly, these findings represent somewhat of a double negative i.e., a model capturing baroreflex resetting is used to demonstrate how a baroreflex that resets could play a role in long-term changes in pressure. Therefore, we have shown that the fact that the baroflex resets, does not mean that the baroreflex cannot play a role in long-term pressure control. Therefore we are confident that it is appropriate to state that we have constructed a model with the goal of "probing hypotheses regarding the long-term control of arterial pressure."

\section{Add the discussion and references of experimental knowledge of baroreflex} stimulation, inhibition, and especially long-term resetting.

Data in Figures 2 and 3 represent direct experimental interrogation of baroreflex stimulation, inhibition, and long-term resetting. The resetting phenomenon is also indirectly probed in Figure 6 where, over the time course of the experiment, the baroreflex response to the acute pressure increase gradually diminishes. The phenomenon and experimental observations are discussed in association with our simulations of these experiments. These are also discussed in the section on arterial stiffening and the etiology of hypertension. Finally, we have added a couple of sentences (end of Results Section 5) comparing our model predictions with the long-term observations of Thrasher on baroreceptor unloading.

Describe how Guyton's models incorporate neural control and explain the above finding, especially the effects of chronic stimulation and how it is different from the proposed model...

A discussion on this point has been added to the section "Results 4".

Discuss existing knowledge or a possible physiological explanation for the finding that 
renin activity will be diminished with baroreflex stimulation.

It is not our finding that renin activity will diminish with baroreflex stimulation. That is the prior experimental observation. The mechanism in the model is through the observed dependence of renin release on sympathetic tone. At a deeper level, the mechanism may simply be explained as follows: decreased sympathetic tone leads to decreased renal arterial tone, leading to increased glomerular filtration and/or medullary blood flow, leading to increased flux of sodium to the macula densa, leading to decreased renin production. While we would rather leave such speculation out of the manuscript, it is published here as part of the open review process.

Discuss what other important physiological mechanisms are not reflected in the model and how they may play a role... explain why macula densa sodium delivery is thought not to be important enough to include in the model, despite the fact that it is known to be one of the key regulators of renin activity.

It is not that we believe that "macula densa sodium delivery is...not...important enough to include in the model." Rather the phenomenon is captured in a simplified way. To clarify this point, we have added the following text to the section on "Neurohumoral control of pressure-diuresis/natriuresis":

"These expressions simplify the known (and unknown) mechanisms governing renin production into a simple phenomenological relationship between pressure, sympathetic tone, and the kinetics of the renin-angiotensin system."

A more biophysically detailed model would be required to represent the direct link between sodium transport and renin production. At the bare minimum, such a model would represent the biophysical processes linking pressure and solute transport in the kidney, including effects of sympathetic innervation on arterial smooth muscle stress development, mechanics of blood and filtrate flow, solute advection, diffusion, permeation, exchange, and pumping.

Do not confuse the model's predictions with its assumptions when discussing simulation results... Conclusion 1 in "Summary and Conclusions" is inappropriate. The fact that the model's assumptions allow for physiologically plausible simulations merely supports the notion that baroreflex may be important in the way the authors have described it.

We agree that this first stated conclusion does not represent a conclusion and what we are trying to say is now better captured in the revised text for Conclusion 1.

This same sentiment applies to the other conclusions, which are theoretical. For example, we conclude that we have demonstrated that "that hypertension does not necessarily indicate any degree of renal dysfunction." It may seem like an equivocation to insert the word "necessarily". However, the literature is full of claims that due to the phenomenon of pressure-diuresis, hypertension necessarily does indicate some degree of renal dysfunction. Our theoretical analysis however would disprove that widespread claim. 
Provide motivation for the chosen elements of the model and outline the model in its entirety first. For example, a flowchart or a diagram of the model may help readers to grasp the overall idea.

We have added a diagram illustrating the control processes captured by the model. (Figure 4B.)

Add at least a basic analysis of parameter sensitivity, with clear description in the Methods section.

This is an excellent idea. A detailed sensitivity analysis is not feasible because the model is expensive to simulate (particularly for the long time frames of Figure 9, for example), the number of parameters is large and would require calculation of $46 \times 46$ Fischer Information matrix (or cross-correlation matrix), and we do not have standard error estimates for all of the data used to identify the model. However, as suggested we have done a basic analysis, and reported a sensitivity index in Table 1. Results are discussed in a new section on parameter sensitivity.

(Also, the sensitivity analysis facilitated as a slightly better parameterization of the model. The results have change imperceptibly.)

In Table 1 please provide physiologically-based description of parameters rather than referring to the equations where they are used... Otherwise, the definitions are circular: parameters are used in the equations without an explanation of their meaning in either the text or the table.

We believe that it useful to the reader to indicate in the table where the parameter is used in the model. Therefore, the indication of which equations given parameters appear in has been retained in the table. In addition, descriptions of the meaning of all parameters have been added wherever they were missing in the text.

Competing Interests: No competing interests were disclosed. 
The benefits of publishing with F1000Research:

- Your article is published within days, with no editorial bias

- You can publish traditional articles, null/negative results, case reports, data notes and more

- The peer review process is transparent and collaborative

- Your article is indexed in PubMed after passing peer review

- Dedicated customer support at every stage

For pre-submission enquiries, contact research@f1000.com 